\title{
Weighted Time-Band Approximation Model for Flight Operations Recovery considering Simplex Group Cycle Approaches in China
}

\author{
Haiwen $X u^{1,2}$ and Songchen $\operatorname{Han}^{3}$ \\ ${ }^{1}$ School of Civil Aviation, Nanjing University of Aeronautics and Astronautics, Nanjing 210016, China \\ ${ }^{2}$ School of Computer Science, Civil Aviation Flight University of China, Guanghan 618307, China \\ ${ }^{3}$ School of Aeronautics \& Astronautics, Sichuan University, Chengdu 610065, China \\ Correspondence should be addressed to Songchen Han; hansongchen001@sina.com
}

Received 15 April 2016; Revised 30 July 2016; Accepted 18 September 2016

Academic Editor: Leonid Shaikhet

Copyright (C) 2016 H. Xu and S. Han. This is an open access article distributed under the Creative Commons Attribution License, which permits unrestricted use, distribution, and reproduction in any medium, provided the original work is properly cited.

\begin{abstract}
The time-band approximation model for flight operations recovery following disruption (Bard, Yu, Arguello, IIE Transactions, 33, 931-947, 2001) is constructed by partitioning the recovery period into time bands and by approximating the delay costs associated with the possible flight connections. However, for disruptions occurring in a hub-and-spoke network, a large number of possible flight connections are constructed throughout the entire flight schedule, so as to obtain the approximate optimal. In this paper, we show the application of the simplex group cycle approach to hub-and-spoke airlines in China, along with the related weighted threshold necessary for controlling the computation time and the flight disruption scope and depth. Subsequently, we present the weighted time-band approximation model for flight operations recovery, which incorporates the simplex group cycle approach. Simple numerical experiments using actual data from Air China show that the weighted time-band approximation model is feasible, and the results of stochastic experiments using actual data from Sichuan Airlines show that the flight disruption and computation time are controlled by the airline operations control center, which aims to achieve a balance between the flight disruption scope and depth, computation time, and recovery value.
\end{abstract}

\section{Introduction}

Flight plans are very important for all airlines, as the efficiency of their construction can significantly affect profit margins and because they involve aircraft, crew, airports, and other important factors. Flight plans contain a series of flight schedules, which consist of the originating city, the departure time, the destination city, and the arrival time for flights the airline intends to operate. However, aircraft mechanical problems, crew unavailability due to illness, and inclement weather conditions often lead to disruptions and delay flight plan propagation [1]. With the rapid increase in scheduled flights and airlines in China, a greater number of airline schedule disruptions are occurring. Moreover, the aircraft, crew, airports, airspace ports, and airspace capacities are connected and restricted; thus, disruptions can lead to a domino effect that generates further flight disruptions. Such disruptions often result in irregular operations such as delays, cancellations, and aircraft reassignment. The majority of irregular operations can be attributed to four major factors, namely, shortage of airline resources (such as crew, aircraft, ground personnel, and gates), airport and airspace capacity shortages, inclement weather conditions, and managementlevel discontent that affects the related resources. In 2013, the economic losses due to airline schedule disruptions exceeded RMB43 billion, according to the Civil Aviation Resource Net of China. However, the Civil Aviation Development Statistical Bulletin reports that, at most, $50.49 \%$ of the schedule disruptions in 2014 can be attributed to airlines, with $26.41 \%$ being the average result. Therefore, the speed with which flight operations are recovered is very important in order to reduce economic losses due to schedule disruptions.

A large amount of research has been devoted to this topic. For example, Teodorović and Guberinić [2] have presented a 
network (representing an airline network) in which the nodes represent the flights and the arcs are the total time losses on individual flights. Hence, a branch and bound procedure was developed to minimize the total passenger delay when an aircraft is grounded. Further, Teodorović and Stojković [3] have introduced a lexicographic dynamic programming approach and a heuristic algorithm to minimize the total passenger delays and the number of cancelled flights. Two minimumcost network flow models to address aircraft shortages have also been introduced by Jarrah et al. [4]. The first is a delay model that allows for flights to be delayed or cancelled, aircraft to be alternated among scheduled flights, or the usage of surplus aircraft to be requested. The second model minimizes the total flight leg cancellation costs by similarly selecting multiple cancellations, employing aircraft substitution, and utilizing spare aircraft. However, accurate flight delay costs can not yet be quickly and easily determined, and the scope and depth of the flight disruption are not considered in the above models. In contrast, delay costs can be approximated with accuracy using the time-band approximation model introduced by $\mathrm{Yu}$ et al., which was developed in order to retain the superiority of the resource assignment model and the multicommodity flow model. Further, Yu [5] has introduced a time-based two-commodity network optimization model for aircraft routing. One commodity in the network model represents aircraft, while the other represents flight cancellations. In addition, Yan and Yang [6] have published a single model to initially combine flight cancellations and delays and to ferry flights. Using a basic time-space network representation, Yan and Lin [7] have developed network flow models to handle station closures, while Yan and $\mathrm{Tu}$ [8] have proposed a multiple-fleet network flow model. Finally, Cao and Kanafani $[9,10]$ have developed a minimum-cost network flow aircraft recovery model, along with network flow algorithms to determine solutions.

However, the mathematical solution derived from a timebased network does not represent the delay costs accurately. In order to develop an optimization model that can determine delay costs with greater accuracy than the multicommodity flow model, the time-band optimization model for aircraft routing reconstruction in response to groundings and delays experienced over the course of a day has been introduced $[11,12]$. A mathematical model that captures the delay costs for each flight based on a time-based network has been developed; the motivation for this approach is to exploit the underlying network structure associated with aircraft routings, with the mathematical model being a single-commodity integral minimum-cost flow problem with side constraints. The concept behind this model is to partition the time horizon into time bands with a fixed length. The nodes in the resulting network represent possible activity at a station. Thus, in the established network, some nodes are positioned at fixed intervals on the time axis. The aggregation of activities for that station can then be attained in $O(m n t)$ operations, where $m$ is the number of stations, $n$ is the number of flights, and

$$
t=\frac{\text { time horizon }}{\text { time segment length }} \text {. }
$$

All aircraft that arrive at a station within the time period represented by a node may then be connected to any flights scheduled to depart from that station. The delay time can be approximated with an accuracy that is dependent on the length of the time segment; therefore, the delay costs can be determined quickly.

When disruptions occur, the airline controllers in the airline operations control centers (AOCC) adjust the scheduled operations by delaying flight departures, cancelling flight legs, rerouting or swapping aircraft, summoning new crew (or reassigning existing crew), and so forth. In order to reassign resources and adjust flight schedules in the most economic manner, certain factors must be acknowledged: the flight delay costs, flight cancellation costs, aircraft substitution costs, aircraft assignment, crew schedules, maintenance requirements, aircraft positions, and so forth. However, it is difficult to determine the delay costs exactly. Further, flight cancellation costs involve both passenger opportunity costs and direct costs, and the delay time cannot be determined in advance. Therefore, the ability to compute both delay and flight cancellation costs is very important as regards the solution of flight operations recovery problems. Such solutions determine adjusted flight departure times, cancellations, and revised routing for affected aircraft.

Many researchers have conducted significant work with regard to flight operations recovery considering passenger factors. For example, Bratu and Barnhart [13] have proposed two flight operations recovery optimization models, the Disrupted Passenger Metric (DPM) and the Passenger Delay Metric (PDM) models, the objective of which is to find the optimal trade-off between airline operation costs and passenger delay costs. The resultant recovery plans work to select flight departure times and flight cancellations, to assign aircraft, to reserve crews for each flight leg as necessary, and to ensure the constraints regarding crew regulations and aircraft maintenance requirements are considered. The objective of the DPM model is to minimize the sum of the operating and disrupted passenger costs. In contrast, the PDM model is constructed to more accurately compute delay costs. Thus, a special procedure to compute the costs due to disrupted passengers has been developed. In addition, $\mathrm{Hu}$ et al. [14] have proposed the integrated integer programming model, which is based on an approximate reduced time-band network and a passenger transiting relationship. The objective is to minimize the total cost associated with reassigning aircraft and passengers to flights. A feasibility analysis for the problem is conducted in order to obtain the conditions under which aircraft and passenger recovery is possible. Further, Zhao et al. [15] have introduced an approach in which the flight delay costs are subdivided, proposing the concept of passenger disappointment spillover cost, and demonstrated a mathematical model of an irregular flight schedule recovery problem that reschedules flight legs and reroutes aircraft by minimizing an objective function involving the flight delay costs or the delay time. The airline can choose one of selections of objective functions according to their needs. However, determining the passenger disappointment spillover cost function remains a problem, and the compensation provided by the airline is a very important 
factor influencing this cost function. For further research on recovery considering passenger factors, see [16-19]. Castro et al. [19] give a new approach for disruption management in airline operations control. They provide a Multiagent System Architecture that represents the AOCC, and introduce a method to calculate the quality costs from the passenger point of view and a system classification to classify disruption management systems. They have done much original work in the recovery operational costs; especially they first propose the quality operational costs (the cost that each minute of delay and flight cancellation has from a passengers point of view). They try to quantify in a monetary unit the cost that each minute of delay and flight cancellation has from a passengers point of view and, at the same time, use that value together with the direct operational costs to improve the decision making during the disruption management process. The quality costs of a flight are given out under the passenger goodwill; what is more, they propose the airline company case study of TAP Portugal, regarding the delay cost from the point of view of the passengers. However, we can not directly use a similar method to calculate the passenger recovery costs in China, in that the preconception of passenger's compensation costs comes from Confucius's idea of the average distribution two thousand years ago and some exemption rules from Civil Aviation Administration of China, such as the weather and other objective reasons, and the passengers can not get any compensation.

When one or several aircraft are grounded, a heuristic approach based on a greedy randomized adaptive search procedure (GRASP) for the reconstruction of aircraft routes, which has been developed by Argüello et al. [20], can be applied. (Further optimization methods are provided in [2125].) However, a large proportion of the feasible optimization methods copy flights to the entire flight schedule, while other resources are reassigned in order to repair the disrupted schedules in the best possible manner; thus, the disruption scope or depth may be wide or great. Further, the computation time increases with increases in the number of potentially disrupted flights, when such disruptions occur in hub-and-spoke airline networks.

Petersen et al. [26] have introduced a disruptable flight set in an attempt to control the recovery search scope of an optimization approach to airline integrated recovery. This approach yields the first computed results for the fully integrated problem. In fact, the disruptable flight set may be comprised of all flights and the actual scope and depth of the disruption may not be controlled. In addition, Lan et al. [27] have introduced aircraft routing and flight departure time adjustment in order to reduce the number of disrupted passengers and to minimize the number of passenger misconnections. However, some passengers may have a long delay time or the flight scope may be wide. The computation time and the disruption scope and depth are not yet fully controllable.

At the 2012 Informs meeting, we first proposed the simplex group cycle approach as a means of controlling the potential disruption scope [28]. The approximated delay costs considering the random flying time around the planned flying time is first introduced in the literature [29], and the time-band approximation model on flight operations recovery problems is constructed based on the time-band network and approximated delay costs. Further, a series of numerical experiments show that the time-band approximation model on flight operations recovery with the random flying time may be more efficient than the time-band approximation model on flight operations recovery with the planned flying time. Using the thought of stochastic delay costs, the approximated cancellation costs formula is first introduced in this paper. In addition, the formula takes into account interval random number, the number of passengers, and approximate city accommodation costs. However, we did not introduce the feasible algorithm for aircraft and flight partitioning, the feasible algorithm to compute weighted value and simulation tests, and so forth, in our previous work.

Previously, Clausen et al. [30] have shown that robust planning or schedule robustness is an interesting research topic closely related to disruption management. The goal of robust planning is to reduce the sensitivity of flight and crew schedules and aircraft rotations to disruptions. Rosenberger et al. [31] have also proposed a robust fleet-assignment model with hub isolation and short cycles and proven that fleet assignments with limited hub connectivity have a greater number of short cycles. A cycle is a sequence of flights that begins and ends at the same airport; further when a cycle contains fewer flights, this cycle is called short cycle. And it is possible to reduce the impact of a disruption at one hub on other hubs by isolating the hubs insofar as possible; hub isolation is zero hub connectivity which is the number of legs in a rotation that are in a route that begins at a hub and ends at a different hub. In fact, the aim of hub isolation and short cycles is to control the difficulty and scope of schedule recovery. However, limited hub isolation corresponds to a lower number of feasible schedule recovery solutions, while short cycles are associated with flight cancellations or longer delay times within the short cycles. The scope of schedule recovery is controlled within the short cycle, rather than by the AOCC controllers.

Moreover, airline network structure has an important effect on flight operations recovery. In America, the AOCC controllers formulating flight operations recovery plans must consider passenger itineraries within a hub-and-spoke network structure. A hub-and-spoke network [19] means a system of connections arranged like a chariot wheel, in which all traffic moves along spokes connected to the hub at the center. However, passenger itineraries of domestic flights in China containing a single flight leg are in the majority, and the international flights accounted for about $9 \%$ of all flights in 2015 according to flights data from the Civil Aviation Administration of China. Further, international flights and domestic flights are different in aircraft type and crews, so there is little implication between international flights and domestic flights. Thus, we do not consider passenger itineraries in this study on domestic flights in China. What is more, multi-hub-and-spoke airlines are associated with multibase airlines in China. Therefore, the hub-and-spoke airlines have some advantages as regards options for schedule recovery from irregular operations. 
This article makes three new contributions compared to previous results. First, we describe more specifically the simplex group cycle approach and first present a feasible algorithm for aircraft and flight partitioning and a feasible algorithm to compute weighted value. Second, we use a weighted threshold to control the disruption scope and depth, computation time and recovery value for flight operations recovery for hub-and-spoke airlines. Hence, we describe the weighted time-band approximation model (WTBAM) for flight operations recovery using the simplex group cycle approach, referring to the concept of the time-band approximation model for flight operations recovery [12]. Third, we design some practical numerical experiments and the results demonstrate that use of the WTBAM incorporating the simplex group cycle is reasonable and feasible, and we provide first the feasible method to choose the weighted threshold $\alpha_{0}$. Furthermore, the results verify that the gap between the relaxed weighted time-band approximation model and the weighted time-band approximation model is zero under certain conditions. Finally, the results demonstrate that we can obtain a satisfactory recovery plan considering the flight disruption scope and depth, computation time and recovery value under some appropriate rules.

The remainder of this paper is organized as follows. In Section 2, we introduce some fundamental concepts, including important definitions and key aspects of the time-band approximation model. In Section 3, we present the mathematical formulations of the WTBAM. The model is then applied to actual data from two airlines and the computational results are presented in Section 4. In Section 5, the conclusions are presented with a brief discussion.

\section{Fundamental Concepts}

To construct the WTBAM, we first introduce several fundamental concepts.

2.1. Objective and Constraints. The objective of flight operations recovery is to make decisions that correspond to the lowest-cost response to restoring the disrupted schedule to the planned schedule. In this paper, we firstly consider flight departure delays or flight leg cancellations to repair a flight schedule after a disruption and subsequently assign individual aircraft to a new flight schedule by the method [32]. Moreover, considering the characteristics of multi-hub-andspoke airlines in China, we have no considering maintenance base for the overnight restrictions in the process of assigning individual aircraft to a new flight schedule. In fact, the AOCC controllers customarily give priority to assign the same type aircraft, and in general there are a lot of the same type aircraft in the multi-hub-and-spoke airlines. Further, the different type of aircraft will bring about a series of problems, such as crew, airport, and maintenance; the AOCC controller has to make sure the aircraft routing is feasible. Therefore, the relaxation of maintenance base restrictions is one of the advantages for multi-hub-and-spoke airlines in China.

Note that minimization of weighted delay costs and flight cancellation costs is a common objective. The delay costs include additional fuel expenses, overtime payments, further expenses associated with overtime payments when an aircraft is delayed in an airport, and passenger food expenses. Thus, delay costs are the direct costs incurred by flight delays, rather than associated costs that arise by chance. We consider the different weighted values of these costs in different simplex group cycles. In contrast, flight cancellation costs include loss of profit, passenger food and accommodation expenses, and passenger compensation. And flight cancellation costs were generated by the approximate estimation formula $y=a x+b$ in this research, $a$ is the number of passengers, $x$ is a random number between 80 and 100 and generally includes meals and passenger compensation, and $b$ is the approximate city accommodation cost. Further, the recovery plan must also satisfy the following constraints in order to be considered feasible. First, the turnaround time must be more than the minimum threshold, that is, $40 \mathrm{~min}$. Second, every flight on each aircraft route must depart from the station at which the immediately preceding flight arrived. Finally, the aircraft balance necessary for the flight schedule to be resumed the next day must be achieved.

2.2. Simplex Group Cycle. The network of a multi-hub-andspoke airline is comprised of two or more hub-and-spoke structures. Thus, multi-hub-and-spoke airlines have more maintenance bases and a greater number of feasible recovery plans. In fact, the group of flights between one or more huband-spoke systems in the same airline network is defined as belonging to a simplex group cycle and labelled $S F_{s}$, where $s$ denotes the specific simplex group cycle. Those flights are assigned to certain fixed aircraft, and the originating and destination cities contain one or more hub-and-spoke systems. Thus, each flight belongs to only one $S F_{s}$. According to the definition of a simplex group cycle, a simplex group cycle partition for all aircraft can be determined, with each aircraft being assigned to one of various $S A_{s}$ groupings. The simplex group cycle approach and its superiority are shown in the following example.

The Air China hub-and-spoke systems shown in the following flight schedule are Beijing (PEK), Shanghai (SHA), and Chengdou (CTU). The network comprised of the PEK, SHA, Xian (XIY), and CTU hubs is shown in Figure 1.

The flight schedule and aircraft assignments are shown in Table 1. Thus, we can determine a partition based on the natural aircraft sequence. The first simplex group cycle is the set comprised of CA4193, CA4102, CA1216, CA1215, CA1945, CA1946, CA425, and CA1950, and the related aircraft are $f_{1}$, $f_{2}$, and $f_{3}$. Thus, the second simplex group cycle is the set comprised of CA1203, CA1204, CA4107, CA4108, CA4201, and CA4202, with the related aircraft being $f_{4}$ and $f_{5}$.

The partitioning of the flights or aircraft is usually determined by the AOCC controllers, according to their empirical data and objectives. However, partitioning the flights or aircraft in a manner that establishes the optimal $S F_{s}$ groupings is a difficult problem. Therefore, we first present a feasible algorithm for aircraft and flight partitioning in Algorithm 1.

2.3. Weighted Set and Potential Disruption Set. When disruptions occur in a hub-and-spoke network, they may lead to 


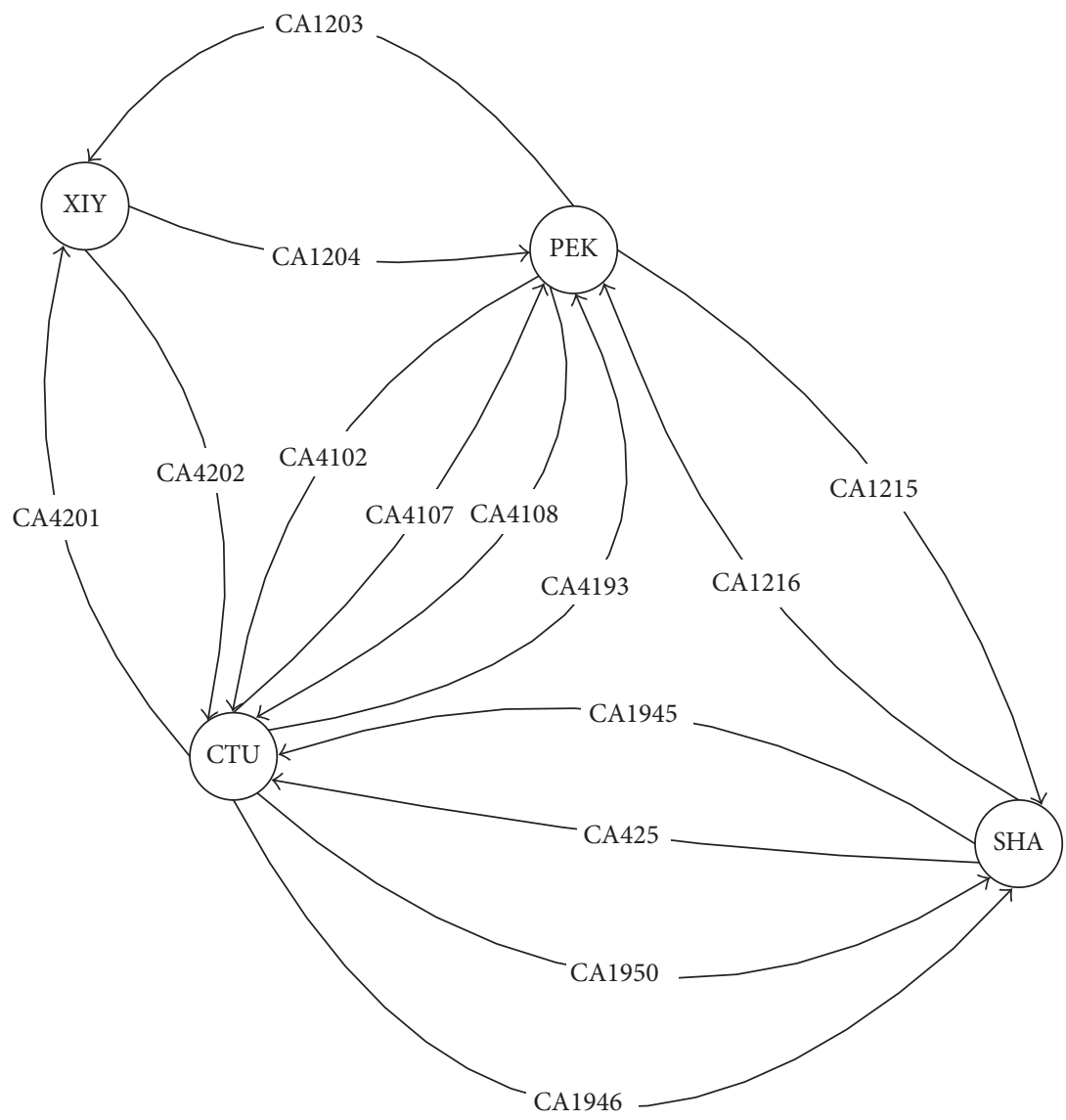

FIGURE 1: Air China flight network comprised of Beijing (PEK), Shanghai (SHA), Xian (XIY), and Chengdou (CTU) hubs.

TABLE 1: Sample flight schedule and aircraft assignments.

\begin{tabular}{|c|c|c|c|c|c|c|c|}
\hline Flight $N$ & Tail $N$ & From & To & Dep. $T$ & Arr. $T$ & Ass. A & C. cost \\
\hline CA4193 & 11 & CTU & PEK & $7: 00$ & $9: 30$ & $f_{1}, 321$ & 11380 \\
\hline CA4102 & 12 & PEK & CTU & $11: 30$ & $14: 00$ & $f_{1}, 321$ & 15150 \\
\hline CA1216 & 21 & SHA & PEK & $8: 00$ & $10: 00$ & $f_{2}, 321$ & 12985 \\
\hline CA1215 & 22 & PEK & SHA & $12: 30$ & $14: 30$ & $f_{2}, 321$ & 16991 \\
\hline CA1945 & 31 & SHA & CTU & 8:05 & $10: 35$ & $f_{3}, 321$ & 11260 \\
\hline CA1946 & 32 & CTU & SHA & $12: 20$ & $14: 50$ & $f_{3}, 321$ & 15785 \\
\hline CA425 & 33 & SHA & CTU & $17: 35$ & $20: 05$ & $f_{3}, 321$ & 13031 \\
\hline CA1950 & 34 & CTU & SHA & $21: 00$ & $23: 30$ & $f_{3}, 321$ & 13820 \\
\hline CA1203 & 41 & PEK & XIY & 07:05 & 9:00 & $f_{4}, 321$ & 11650 \\
\hline CA1204 & 42 & XIY & PEK & $10: 00$ & 11:55 & $f_{4}, 321$ & 10256 \\
\hline CA4107 & 51 & CTU & PEK & 09:00 & $11: 30$ & $f_{5}, 321$ & 11000 \\
\hline CA4108 & 52 & PEK & CTU & $15: 00$ & $17: 30$ & $f_{5}, 321$ & 15236 \\
\hline CA4201 & 53 & CTU & XIA & $20: 45$ & $22: 00$ & $f_{5}, 321$ & 9325 \\
\hline CA4202 & 54 & XIA & CTU & 23:00 & 00:15 & $f_{5}, 321$ & 9128 \\
\hline
\end{tabular}

Flight N: flight number; Tail N: tail number; Dep. T: departure time; Arr. T: arrival time; Ass. A: assigned aircraft; C. cost: cancellation cost.

a large amount of disruptions overall. Thus, schedule recovery should be accomplished in the most economic manner. However, if we only consider the flights themselves, the disruptions may have a negative influence on passengers. Therefore, in order to control the potential disruption scope
$F_{s}$ in a hub-and-spoke network and the associated negative influence on passengers, $F_{s}$ can be determined based on the flight disruption scope and depth, the computation time, and the recovery value. Therefore, we employ the concept of a weighted set, as explained below. 


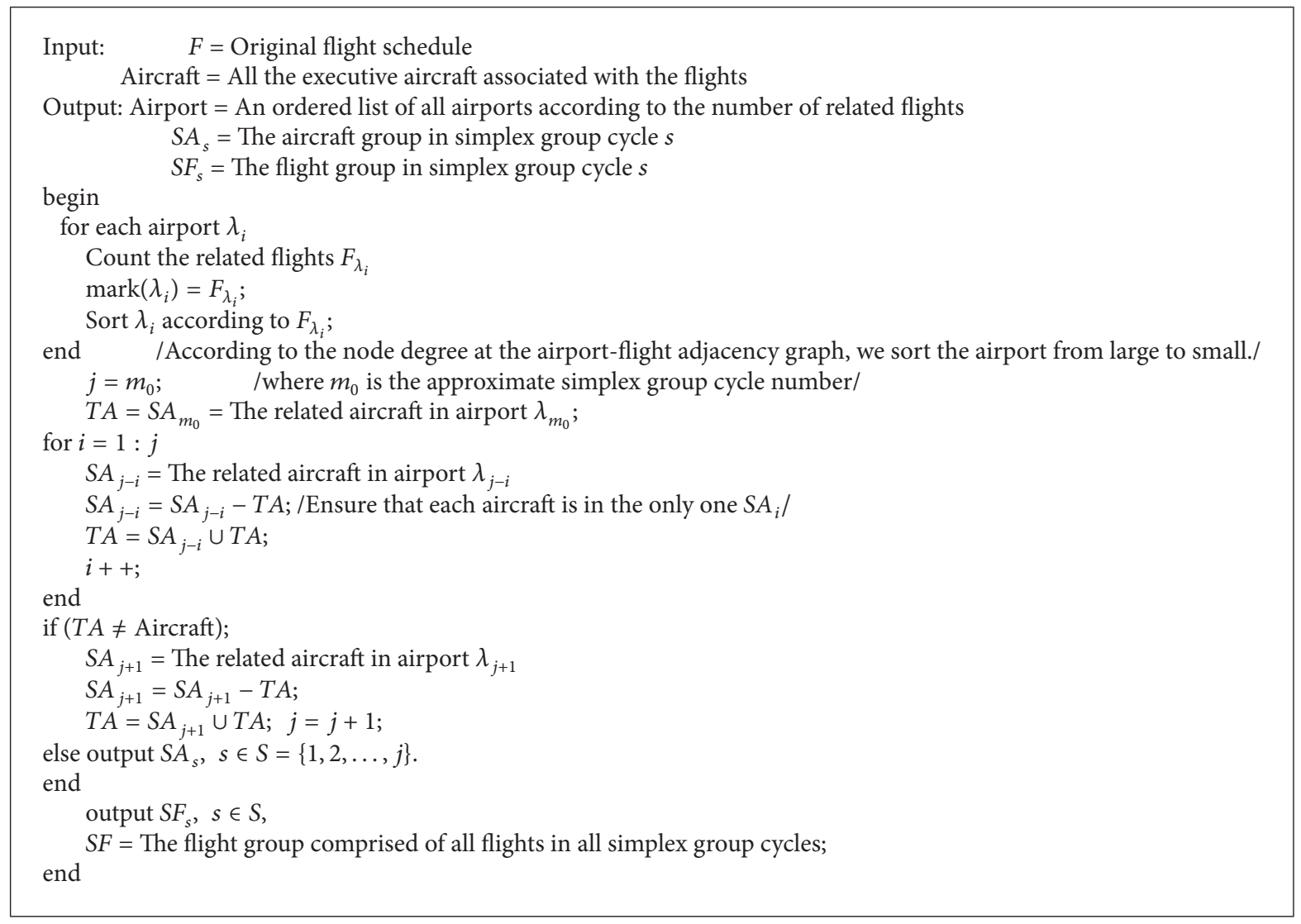

Algorithm 1: Simplex group cycle partitioning (SGCP) algorithm (S Algorithm).

Let $S^{*}$ be the set containing the simplex group cycle $s$ which is at least one schedule disruption in $s \in S$. And let $S A$ be the aircraft partition related to $S$, the set of all simplex group cycles. Then $S A^{*}$ is the aircraft partition related to $S^{*}$.

Definition 1. When a disruption occurs, the weighted value is denoted

$$
w_{j}= \begin{cases}\sum_{i \in S A^{*}} w_{i j}, & j \notin S A^{*}, \\ 1, & j \in S A^{*} .\end{cases}
$$

When the flights of the $i$ th simplex group cycle influence those of the $j$ th simplex group cycle, the value of $w_{i j}$ can usually be assigned by the AOCC controllers in accordance with their empirical data and objectives. Naturally, we define $w_{i i}=1, \forall i \in S$, and $w_{i j}=\beta\left(1+A T_{S A_{j}} / A T_{S A_{i}}\right), \forall i, j \in S$. Here, $\beta$ is the amplification coefficient, and $A T_{S A_{i}}$ and $A T_{S A_{j}}$ are the average available flight times of each $S A_{i}$ and $S A_{j}$, respectively. Then, the weighted set $W=\left\{w_{j}, \forall j \in S\right\}$.

The simplex group cycle approach can exploit the advantages provided by a multi-hub-and-spoke network. For example, in Table 1, when aircraft $f_{1}$ (CA4193) departed from CTU arrived in PEK, the maintenance personnel detected mechanical problems. It was estimated that a period of $4 \mathrm{~h}$ was required to complete the repairs. Thus, using the simplex group cycle approach, we utilize $f_{2}$ to execute flight CA4102, followed by flight CA1950, with $f_{3}$ being utilized for flights CA1945 and CA1946. At 13:30, $f_{1}$ then executes flights CA1215 and CA425. The total delay time is $100 \mathrm{~min}$, and we save $60 \mathrm{~min}$. Note that only the disruption scope is controlled by the simplex group cycle approach. In addition, the developed recovery plan involved cooperation between China Airlines $\left(f_{2}, f_{3}\right)$ and its branch company, China Southwest Airlines $\left(f_{1}\right)$.

Based on a weighted threshold $\alpha_{0}$, AOCC airline controllers can obtain a potential disrupted set $P D F S^{\alpha_{0}}$, which is defined as follows.

Definition 2. The potential disrupted flights set $P D F S^{\alpha_{0}}$ is the set of flights in the simplex group cycle $s$ obtained when the condition $w_{s} \leq \alpha_{0}, \forall s \in S$, is satisfied.

Hence, the AOCC airline controllers can set the potential disruption flights $P D F S^{\alpha_{0}}$ conveniently by considering the value of $\alpha_{0}$. However, it is difficult to compute the $w_{i j}$ values for flights or aircraft. Therefore, a feasible algorithm to compute $W$ and $P D F S^{\alpha_{0}}$ is first given in Algorithm 2. The timeband network incorporating $P D F S^{\alpha_{0}}$ is constructed using the time-band network transformation procedure found in [11, 12]. The time-band network mainly consists of station-time nodes, station-sink nodes, and flight arcs, so we refer to some concepts of the literature $[11,12]$ in the following 


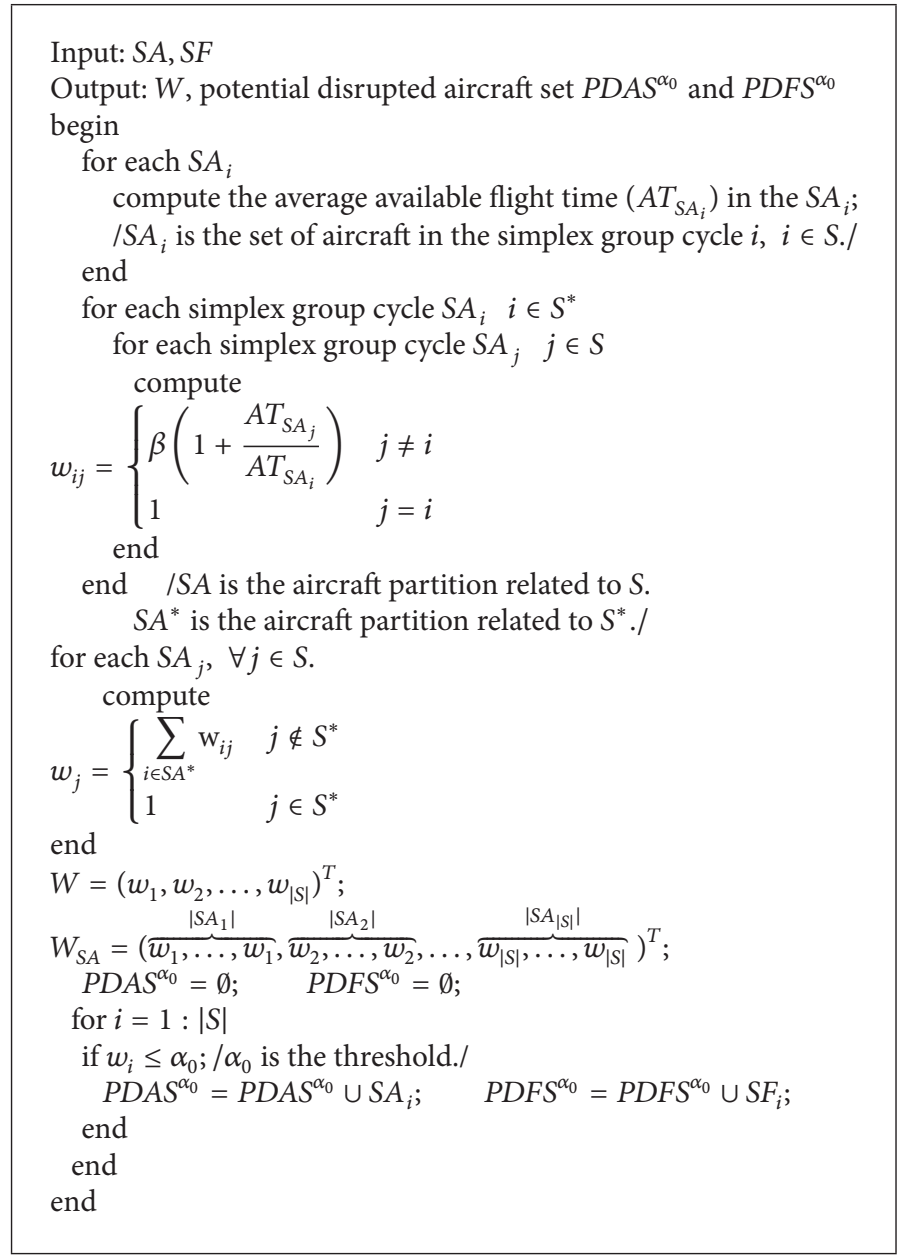

Algorithm 2: Weighted set and potential disruption flights set $\left(P D F S^{\alpha_{0}}\right)$ algorithm (W Algorithm).

Flight arcs: arcs representing specific scheduled flights.

Station-time nodes: nodes representing aggregate activity for a specific time segment at a station.

Station-sink nodes: nodes representing the end of the recovery period at stations.

Subsequently all the possible delay costs for the flight arcs can be computed by considering the difference between the real takeoff time and the scheduled plan in the time-band network. Naturally, the WTBAM is constructed in accordance with the optimization model by considering a series of flight arcs with some potential flight delays. In the next section, we present the mathematical formulation of the time-band approximation model.

\section{Time-Band Approximation Model Mathematical Formulation}

In order to construct the mathematical formulation of the optimization model, some indices, sets, parameters, and variables are employed, which are given in Nomenclature.
Using $\alpha_{0}$ and considering the recovery plan for $P D F S^{\alpha_{0}}$ only, we can express the WTBA approximation model as follows:

$$
\begin{aligned}
\min & F_{\alpha_{0}}(x, y, z) \\
= & \sum_{k \in P D F S^{\alpha_{0}}} \sum_{i \in P(k)} \sum_{j \in H(k, i)} w_{i j}^{k} d_{i j}^{k} x_{i j}^{k} \\
& +\sum_{k \in P D F S^{\alpha_{0}}} w^{k} c_{k} y_{k},
\end{aligned}
$$

$$
\begin{aligned}
& \text { subject to } \sum_{i \in P(k)} \sum_{j \in H(k, i)} x_{i j}^{k}+y_{k}=1, \quad \forall k \in P D F S^{\alpha_{0}}, \\
& \sum_{k \in G(i)} \sum_{j \in H(k, i)} x_{i j}^{k}+z_{i}-\sum_{k \in N(i)} \sum_{j \in M(k, i)} x_{i j}^{k} \\
& =a_{i}^{\alpha_{0}}
\end{aligned}
$$

$\forall i \in I, k \in P D F S^{\alpha_{0}}$,

$\sum_{k \in N(i)} \sum_{j \in M(k, i)} x_{j i}^{k}+\sum_{j \in Q(i)} z_{j}=h_{i}^{\alpha_{0}}$,

$\forall i \in J, k \in P D F S^{\alpha_{0}}$, 


$$
\begin{aligned}
& x_{i j}^{k} \in\{0,1\}, \\
& \quad k \in \operatorname{PDFS}^{\alpha_{0}}, i \in I, \quad j \in H(k, i), \\
& y_{k} \in\{0,1\}, \quad k \in P D F S^{\alpha_{0}}, \\
& z_{i} \in Z_{+}, \quad i \in I .
\end{aligned}
$$

The objective function (3) minimizes the delay and cancellation costs for $P D F S^{\alpha_{0}}$. Constraint (4) shows that all flights in the simplex group cycle must be operated or cancelled. Further, constraint (5) satisfies the aircraft utilization requirement at the station-time node. The station-sink node flow constraint (6) enforces aircraft balance. The binary $x_{i j}^{k}$ and $y_{k}$ ensure that a flight can only be operated or cancelled; that is, it must not be partially serviced and then cancelled. The possible arcs must be integrated, which is enforced by constraint (9).

Remark 3. When $\alpha_{0}=1$, the solution of WTBAM is obtained after searching the flights in $S^{*}$, and the passengers affected by this solution may experience long flights, or some flights in the simplex group cycle may be cancelled. In other words, the disruption scope can be controlled in the simplex group cycle $S^{*}$, but the disruption depth may be very large.

In contrast to the optimization model with $\alpha_{0} \geq 1$, the objective function and constraints in the model can be modified by $k \in S F_{S^{*}}$. In this optimization model, $P D F S^{\alpha_{0}}$ is restricted to the disrupted flight set $S F_{S^{*}}$ only. We can obtain the optimization model with $W_{S^{*}}=1$, as follows:

$$
\begin{aligned}
& \min f_{S^{*}}(x, y, z) \\
& =\sum_{s \in S^{*}} \sum_{k \in S F_{s}}\left(\sum_{i \in P(k)} \sum_{j \in H(k, i)} d_{i j}^{k} x_{i j}^{k}+c_{k} y_{k}\right), \\
& \text { subject to } \sum_{i \in P(k)} \sum_{j \in H(k, i)} x_{i j}^{k}+y_{k}=1, \quad \forall k \in S F_{S^{*}} \text {, } \\
& \sum_{k \in G(i)} \sum_{j \in H(k, i)} x_{i j}^{k}+z_{i}-\sum_{k \in N(i)} \sum_{j \in \mathrm{M}(k, i)} x_{i j}^{k} \\
& =a_{i}^{1}, \\
& \forall i \in I, k \in S F_{S^{*}}, \\
& \sum_{k \in N(i)} \sum_{j \in M(k, i)} x_{j i}^{k}+\sum_{j \in Q(i)} z_{j}=h_{i}^{1} \\
& \forall i \in J, k \in S F_{S^{*}} \text {, } \\
& x_{i j}^{k} \in\{0,1\} \text {, } \\
& k \in S F_{S^{*}}, \quad i \in I, \quad j \in H(k, i), \\
& y_{k} \in\{0,1\}, \quad k \in S F_{S^{*}} \text {, } \\
& z_{i} \in Z_{+}, \quad i \in I \text {. }
\end{aligned}
$$

The objective function (10) minimizes the delay costs and the cancellation costs in $S^{*}$. Further, constraint (11) shows
TABLE 2: Flight schedule and aircraft assignments for $P D F S^{2.0}$.

\begin{tabular}{lcccccc}
\hline Tail $N$ & From & To & Dep. $T$ & Arr. $T$ & Ass. A & C. Cost \\
\hline 12 & PEK & CTU & $11: 30$ & $14: 00$ & $f_{1}, 321$ & 15150 \\
22 & PEK & SHA & $12: 30$ & $14: 30$ & $f_{2}, 321$ & 16991 \\
32 & CTU & SHA & $12: 20$ & $14: 50$ & $f_{3}, 321$ & 15785 \\
33 & SHA & CTU & $17: 35$ & $20: 05$ & $f_{3}, 321$ & 13031 \\
34 & CTU & SHA & $21: 00$ & $23: 30$ & $f_{3}, 321$ & 13820 \\
42 & XIY & PEK & $10: 00$ & $11: 55$ & $f_{4}, 321$ & 10256 \\
52 & PEK & CTU & $15: 00$ & $17: 30$ & $f_{5}, 321$ & 15236 \\
53 & CTU & XIY & $20: 45$ & $22: 00$ & $f_{5}, 321$ & 9325 \\
54 & XIY & CTU & $23: 00$ & $00: 15$ & $f_{5}, 321$ & 9128 \\
\hline
\end{tabular}

TABLE 3: Nonzero flight arc delay costs with disruption threshold $\alpha_{0}=1.0$.

\begin{tabular}{lccc}
\hline Tail $N$ & $\begin{array}{c}\text { Origin } \\
\text { node }\end{array}$ & $\begin{array}{c}\text { Destination } \\
\text { node }\end{array}$ & $\begin{array}{c}\text { Arc delay } \\
\text { cost }\end{array}$ \\
\hline 12 & 3 & 7 & 3000 \\
21 & 3 & 6 & 1800 \\
32 & 4 & 8 & 3200 \\
32 & 7 & 9 & 6200 \\
32 & 10 & 14 & 10400 \\
32 & 11 & 14 & 11600 \\
33 & 8 & 11 & 1100 \\
33 & 9 & 13 & 4100 \\
34 & 11 & 14 & 1200 \\
\hline
\end{tabular}

that all flights in $\bigcup_{s \in S^{*}} S F_{s}$ must be operated or cancelled. Constraint (12) satisfies the aircraft utilization requirement for $\bigcup_{s \in S^{*}} S F_{s}$ at the station-time node. The station-sink node flow constraint (13) enforces aircraft balance in $\bigcup_{s \in S^{*}} S F_{s}$. The binary $x_{i j}^{k}$ and $y_{k}$ ensure that a flight can only be operated or cancelled; that is, it must not be partially serviced and then cancelled. Finally, the copy arcs must be integrated, which is enforced by constraint (16).

Remark 4. When $\alpha_{0}$ has the maximum weighted value $\alpha_{\text {sup }}$, $F_{s}$ corresponds to all of the flights. In that case, it may be very difficult for the AOCC to quickly obtain the optimal solution or the disrupted flight scope may be very broad. It may be very difficult to implement such a recovery plan for actual flight production, because it does not satisfy the requirements of timeliness and balance between the disruption scope, disruption depth, and recovery value.

For $1 \leq \alpha_{0} \leq \alpha_{\text {sup }}$, it is difficult for the AOCC to determine the optimal $\alpha_{0}$ that satisfies the balance between the passenger and airline requirements. However, the AOCC can choose the best satisfactory value under those conditions.

Remark 5. When a disruption occurs, the weighted value is given out by the $W$ Algorithm, Then

$$
w_{i j}^{k}=w^{k}=w_{s}
$$

where $\left\{w_{s} \mid \exists s \in S\right.$, s.t. $\left.k \in P D F S^{\alpha_{\text {sup }}}\right\}$. 
TABLE 4: Nonzero flight arc delay costs with disruption threshold $\alpha_{0}=2.0$.

\begin{tabular}{|c|c|c|c|}
\hline Tail $N$ & $\begin{array}{c}\text { Origin } \\
\text { node }\end{array}$ & $\begin{array}{c}\text { Destination } \\
\text { node }\end{array}$ & $\begin{array}{c}w_{i j}^{k} * \text { arc } \\
\text { delay cost }\end{array}$ \\
\hline 12 & 4 & 10 & 1200 \\
\hline 12 & 5 & 11 & 1800 \\
\hline 12 & 6 & 13 & 1800 \\
\hline 22 & 5 & 9 & 600 \\
\hline 22 & 6 & 12 & 3420 \\
\hline 32 & 7 & 14 & 3200 \\
\hline 32 & 10 & 16 & 4400 \\
\hline 32 & 11 & 17 & 5000 \\
\hline 32 & 13 & 18 & 6200 \\
\hline 32 & 15 & 20 & 7400 \\
\hline 32 & 19 & 27 & 10400 \\
\hline 32 & 21 & 27 & 11600 \\
\hline 32 & 22 & 27 & 12800 \\
\hline 32 & 24 & 27 & 14000 \\
\hline 33 & 14 & 21 & 1100 \\
\hline 33 & 16 & 22 & 2300 \\
\hline 33 & 17 & 24 & 2900 \\
\hline 33 & 18 & 26 & 4100 \\
\hline 33 & 20 & 29 & 5300 \\
\hline 34 & 21 & 27 & 1200 \\
\hline 34 & 22 & 27 & 2400 \\
\hline 34 & 24 & 27 & 3600 \\
\hline 42 & 23 & 28 & 29640 \\
\hline 42 & 25 & 28 & 31920 \\
\hline 53 & 19 & 23 & 570 \\
\hline 53 & 21 & 25 & 1800 \\
\hline 53 & 22 & 30 & 5130 \\
\hline 53 & 24 & 30 & 6270 \\
\hline 53 & 26 & 30 & 8550 \\
\hline 54 & 25 & 29 & 2280 \\
\hline
\end{tabular}

Proof. Because all the flights are contained within $P D F S^{\alpha_{\text {sup }}}=$ $\bigcup_{s \in S} S F_{s}$, when $i \neq j, S F_{i} \neq S F_{j}, \forall i, j \in S$. Therefore, we can obtain

$$
\exists s \in S \text {, s.t. } k \in P D F S^{\alpha_{\text {sup }}} \text {. }
$$

In fact, $z_{i}$ must be less than or equal to the total number of aircraft $\sum_{s \in S}\left|S A_{s}\right|$. And then the linear programming relaxation of the weighted time-band approximation model (LPRWTBAM) is expressed as follows:

$$
\begin{aligned}
\min & f_{\alpha_{0}}(x, y, z) \\
& =\sum_{k \in P D F S^{\alpha_{0}}}\left(\sum_{i \in P(k)} \sum_{j \in H(k, i)} w_{i j}^{k} d_{i j}^{k} x_{i j}^{k}+w^{k} c_{k} y_{k}\right),
\end{aligned}
$$

$$
\begin{gathered}
\text { subject to } \sum_{i \in P(k)} \sum_{j \in H(k, i)} x_{i j}^{k}+y_{k}=1, \quad \forall k \in P D F S^{\alpha_{0}}, \quad(20) \\
\sum_{k \in G(i)} \sum_{j \in H(k, i)} x_{i j}^{k}+z_{i}-\sum_{k \in N(i)} \sum_{j \in M(k, i)} x_{i j}^{k}=a_{i}^{\alpha_{0},} \\
\forall i \in I, k \in P D F S^{\alpha_{0}}, \\
\sum_{k \in N(i)} \sum_{j \in M(k, i)} x_{j i}^{k}+\sum_{j \in Q(i)} z_{j}=h_{i}^{\alpha_{0}}, \\
x_{i j}^{k} \in[0,1], \quad k \in P D F S^{\alpha_{0}}, i \in I, \quad j \in H(k, i), \\
y_{k} \in[0,1], \quad k \in P D F S^{\alpha_{0}}, \\
z_{i} \in\left[0, \sum_{s \in S}\left|S A_{s}\right|\right], \quad i \in I .
\end{gathered}
$$

Naturally, $w_{i j}^{k} d_{i j}^{k} \geq 0$ and $w^{k} c_{k} \geq 0, \forall i \in I, j \in H(k, i)$, $k \in P D F S^{\alpha_{0}}$. We can set

$$
\begin{aligned}
& \left(I_{0}, J_{0}, K_{0}\right)=\left\{\left(i_{0}, j_{0}, k_{0}\right) \mid w_{i_{0} j_{0}}^{k_{0}} d_{i_{0} j_{0}}^{k_{0}}>0, i_{0} \in I, j_{0}\right. \\
& \left.\quad \in H\left(k_{0}, i_{0}\right)\right\}, \quad k_{0} \in P D F S^{\alpha_{0}}, \\
& K_{1}=\left\{k_{1} \mid w^{k_{1}} c_{k_{1}}>0, k_{1} \in P D F S^{\alpha_{0}}\right\} .
\end{aligned}
$$

Further, we assume that $w_{i j}^{k} d_{i j}^{k}$ and $w^{k} c_{k}$ satisfy the following properties.

Well-Ordered Property. The nonzero value coefficient set (NVCS)

$$
\left\{w_{i j}^{k} d_{i j}^{k}, \forall(i, j, k) \in\left(I_{0}, J_{0}, K_{0}\right)\right\} \cup\left\{w^{k} c_{k}, \forall k \in K_{1}\right\}
$$

is well ordered according to $<$.

In fact, the weighted value, delayed time, and cancellation cost are often different in the NVCS; therefore, the following sequence properties may be satisfied in most cases

$$
\begin{aligned}
w_{00}^{0} d_{00}^{0} & <w_{00}^{0} d_{00}^{1}<\cdots<w_{01}^{0} d_{01}^{0}<w_{01}^{1} d_{01}^{1}<\cdots \\
& <w_{\left|I_{0}\right|\left|J_{0}\right|}^{\left|K_{0}\right|} d_{\left|I_{0}\right|\left|J_{0}\right|}^{\left|K_{0}\right|} \quad(i, j, k) \in\left(I_{0}, J_{0}, K_{0}\right), \\
w^{0} c_{0} & <w^{1} c_{1}<\cdots<w^{\left|K_{1}\right|} c_{\left|K_{1}\right|}, \quad k \in K_{1} .
\end{aligned}
$$

Naturally, the NVCS frequently exhibits a well-ordered property.

Let $\left\|F_{0}^{k}\right\|$ be the number of elements in the $F_{0}^{k}$ set. Then, we can obtain the following property in the majority of cases.

Possible Zero Property. $\left\|F_{0}^{k}\right\|=0$ or $1, F_{0}^{k}=\left\{(i, j, k) \mid w_{i j}^{k} d_{i j}^{k}=\right.$ $0\}, \forall k \in P D F S^{\alpha_{0}}$. In fact, when there is only a small number of available aircraft in $i \in P(k)$, the aircraft utilization is generally reasonably high and flights cannot take off early; then $\left\|F_{0}^{k}\right\|=0$ or 1 often occurs.

Remark 6. If the feasible solution of WTBAM is nonempty, $a_{i}^{\alpha_{0}}$ and $h_{i}^{\alpha_{0}}$ are integers, and NVCS exhibits a well-ordered 
TABLE 5: Results for $\alpha_{0}=1.0$

\begin{tabular}{|c|c|c|c|c|c|c|c|}
\hline \multicolumn{8}{|c|}{$\begin{array}{c}\min z=15785 \\
h_{1}=h_{2}=h_{3}=1\end{array}$} \\
\hline Tail $N$ & From & To & OST node & DST node & Ass. A & D. cost & C. cost \\
\hline 12 & PEK & CTU & 2 & 4 & $f_{2}$ & 0 & - \\
\hline 21 & PEK & SHA & 1 & 5 & $f_{3}$ & 0 & - \\
\hline 32 & CTU & SHA & - & - & - & - & 15758 \\
\hline 33 & SHA & CTU & 5 & 10 & $f_{3}$ & 0 & - \\
\hline 34 & CTU & SHA & 4 & 14 & $f_{2}$ & 0 & - \\
\hline
\end{tabular}

OST: origin station-time; DST: destination station-time; D. cost: delay cost.

TABLE 6: Results for $\alpha_{0}=2.0$.

\begin{tabular}{lccccccc}
\hline \multicolumn{7}{c}{$\min z=0$} \\
\multicolumn{7}{c}{$h_{1}=2, h_{2}=1, h_{3}=2, h_{4}=0$} \\
\hline Tail $N$ & From & To & OST node & DST node & Ass. A & D. cost & C. cost \\
\hline 12 & PEK & CTU & 2 & 7 & $f_{2}$ & 0 & - \\
22 & PEK & SHA & 4 & 8 & $f_{5}$ & 0 & - \\
32 & CTU & SHA & 3 & 8 & $f_{3}$ & 0 & - \\
33 & SHA & CTU & 8 & 19 & $f_{3}$ & 0 & - \\
34 & CTU & SHA & 19 & 27 & $f_{1}$ & 0 & - \\
42 & XIY & PEK & 1 & 5 & $f_{4}$ & 0 & - \\
52 & PEK & CTU & 6 & 15 & $f_{1}$ & 0 & - \\
53 & CTU & XIY & 15 & 23 & $f_{2}$ & 0 & - \\
54 & XIY & CTU & 23 & 29 & $f_{2}$ & 0 & - \\
\hline
\end{tabular}

TABLE 7: First simplex group cycle partition.

\begin{tabular}{lccccccccccccc}
\hline \multicolumn{11}{l|}{ SGC $N$} & \multicolumn{11}{c}{ Tail $N$} \\
\hline$S_{1}$ & 16 & 17 & 18 & 21 & 31 & 32 & 33 & 35 & 39 & 42 & & & \\
$S_{2}$ & 1 & 22 & 25 & 26 & 27 & 30 & 38 & 48 & 52 & 54 & 55 & 57 & 60 \\
$S_{3}$ & 2 & 3 & 8 & 9 & 10 & 11 & 34 & 40 & 41 & 43 & 44 & & \\
$S_{4}$ & 29 & 36 & 37 & 47 & 49 & 53 & & & & & & \\
$S_{5}$ & 5 & 6 & 15 & 20 & 56 & 58 & 59 & & & & & & \\
$S_{6}$ & 4 & 7 & 12 & 19 & 23 & 24 & 46 & & & & & & \\
$S_{7}$ & 13 & 14 & 28 & 45 & 50 & 51 & & & & & & & \\
\hline
\end{tabular}

SGC N: Simplex group cycle number.

property, then $F_{0}^{k}$ has the possible zero property, $\forall k \in$ $P D F S^{\alpha_{0}}, i \in P(k)$. Then, the WTBAM and LPRWTBAM solutions are identical.

Proof. Let $\left(x_{l p}^{*}, y_{l p}^{*}, z_{l p}^{*}\right)^{T}$ be the LPRWTBAM solution and $\left(x^{*}, y^{*}, z^{*}\right)^{T}$ be the WTBAM solution. Then, because the LPRWTBAM is the relaxed WTBAM,

$$
f\left(x_{l p}^{*}, y_{l p}^{*}, z_{l p}^{*}\right) \leq f\left(x^{*}, y^{*}, z^{*}\right) .
$$

If $\left(x_{l p}^{*}, y_{l p}^{*}, z_{l p}^{*}\right)^{T}$ is an integer, then

$$
\left(x^{*}, y^{*}, z^{*}\right)^{T}=\left(x_{l p}^{*}, y_{l p}^{*}, z_{l p}^{*}\right)^{T} .
$$

The analysis of $\left(x_{l p}^{*}, y_{l p}^{*}, z_{l p}^{*}\right)^{T}$ is as follows.
TABLE 8: Second simplex group cycle partition.

\begin{tabular}{lcccccccc}
\hline SGC $N$ & \multicolumn{1}{c}{ Tail $N$} \\
\hline$S_{1}$ & 17 & 18 & 32 & 35 & 39 & 42 & & \\
$S_{2}$ & 22 & 25 & 26 & 38 & 57 & & & \\
$S_{3}$ & 2 & 8 & 9 & 10 & 11 & 34 & 40 & 41 \\
$S_{4}$ & 29 & 36 & 37 & 49 & 53 & & & \\
$S_{5}$ & 5 & 6 & 15 & 20 & 56 & 59 & & \\
$S_{6}$ & 12 & 23 & 24 & 46 & & & & \\
$S_{7}$ & 27 & 43 & 52 & 58 & & & & \\
$S_{8}$ & 1 & 30 & 33 & 47 & 48 & & & \\
$S_{9}$ & 13 & 14 & 28 & 45 & 50 & 51 & & \\
$S_{10}$ & 3 & 16 & 44 & 55 & 60 & & & \\
$S_{11}$ & 4 & 19 & 21 & & & & & \\
$S_{12}$ & 7 & 31 & 54 & & & & &
\end{tabular}

TABLE 9: Third simplex group cycle partition.

\begin{tabular}{lcccccccc}
\hline SGC $N$ & \multicolumn{1}{c}{ Tail $N$} \\
\hline$S_{1}$ & 18 & 32 & 35 & 39 & 42 & & & \\
$S_{2}$ & 22 & 25 & 26 & 38 & & & & \\
$S_{3}$ & 2 & 8 & 9 & 10 & 11 & 34 & 40 & 41 \\
$S_{4}$ & 29 & 36 & 37 & 49 & & & & \\
$S_{5}$ & 5 & 6 & 15 & 20 & 56 & 59 & & \\
$S_{6}$ & 23 & 24 & & & & & & \\
$S_{7}$ & 43 & 58 & & & & & & \\
$S_{8}$ & 1 & 30 & 47 & 48 & & & & \\
$S_{9}$ & 14 & 28 & 45 & 51 & & & & \\
$S_{10}$ & 3 & 16 & 44 & 55 & 60 & & & \\
$S_{11}$ & 4 & 19 & 21 & & & & & \\
$S_{12}$ & 7 & 31 & 54 & & & & \\
$S_{13}$ & 12 & 46 & 50 & & & & \\
$S_{14}$ & 13 & 52 & 57 & & & & \\
$S_{15}$ & 15 & 17 & 27 & 53 & & & & \\
\hline
\end{tabular}

When $\left\|F_{0}^{k}\right\|=0$ or $1, \forall k \in P D F S^{\alpha_{0}}$, we adopt the assumption

$$
\exists\left(i, j, k_{0}\right) \text {, s.t. } w_{i j}^{k_{0}} d_{i j}^{k_{0}}=0 .
$$


TABLE 10: The impact of disruption flights scope and depth.

\begin{tabular}{lccccc}
\hline & \multicolumn{5}{c}{ Delay time band } \\
& DT 0 & DT 15 & DT 30 & DT 60 & $\begin{array}{c}\text { Cancelled } \\
\text { flight }\end{array}$ \\
\hline $\begin{array}{l}\text { Weighted } \\
\text { value }\end{array}$ & 1 & 2 & 4 & 6 & 8 \\
\hline
\end{tabular}

Then $w_{i j}^{k_{0}} d_{i j}^{k_{0}} \cup$ NVCS is well ordered. According to $x_{i j}^{k} \in[0,1]$, $y_{k} \in[0,1], z_{i} \in\left[0, M_{0}\right], i \in I,(19)$, and (20), we can obtain

$$
\begin{aligned}
x_{i j}^{k} & =\{0,1\}, \\
y_{k} & =\{0,1\},
\end{aligned}
$$

$$
\forall k \in P D F S^{\alpha_{0}} \text {. }
$$

Furthermore, according to (21) and (22) and as $a_{i}^{\alpha_{0}}$ and $h_{i}^{\alpha_{0}}$ are integers, we know that $z_{i}=\left\{0,1, \ldots, M_{0}\right\}$, where $M_{0}$ is an integer. Finally, we conclude that $\left(x_{l p}^{*}, y_{l p}^{*}, z_{l p}^{*}\right)^{T}$ is an integer.

\section{Computational Results}

Two sets of computational results are presented in this section: (i) the computational results obtained by application of the WTBAM to the sample data given in Table 1 , which demonstrate that use of the WTBAM incorporating the simplex group cycle is reasonable and feasible; (ii) results of a test using data from actual aircraft belonging to Sichuan Airlines in China, which involves a hub-and-spoke network. The analysis results obtained for different weighted thresholds and different simplex group cycles under four scenarios are given.

4.1. Sample Flight Schedule Table 1. Mechanical problems are detected for aircraft $f_{1}$ at 9:50, and it is estimated that a 4-h period is required for the repair work. In this example, the time band is set to half an hour and the schedule recovery is implemented from 10:00 to 02:00 the next day. In this example, the simplex group cycle partitions are set to $S_{1}$ and $S_{2}$, in order to show the AOCC controllability. Note that these partitions do not correspond to the partition implemented using the simplex group cycle procedure. The weighted value $W_{2}=1.9$ is determined according to the weighted set procedure. We set the disruption threshold $\alpha_{0}=2.0$; then the potential disruption flights set $P D F S^{2.0}$ is as shown in Table 2.

Subsequently, the flight arc delay costs for the recovery problem are determined using the time-band transformation network [12]. The time-band transformation contains 26 nodes and 57 flight arcs. The delay cost for each flight arc is calculated according to the arc delay, and a 20-per-min delay cost function is assumed [12] in this paper. The nonzero flight arc delay costs for $\alpha_{0}=1.0$ and 2.0 are listed in Tables 3 and 4 , respectively. Finally, we can compute the WTBAM solution using Lingo 14.0. The solutions for $\alpha_{0}=1.0$ and 2.0 are listed in Tables 5 and 6 , respectively. The results obtained for the time-band transformation with $\alpha_{0}=2.0$ contain more nodes and flight arcs than that for $\alpha_{0}=1.0$; therefore, the WTBAM solution (with $\alpha_{0}=2.0$ ) may yield a superior proximal point than the standard time-band transformation with $\alpha_{0}=1.0$. Further, the optimal value yielded by the WTBAM is smaller than that for the time-band transformation with $\alpha_{0}=1.0$.

4.2. Stochastic Experiments. Our model was also tested using actual data obtained for a specific day in 2015, for a hub-andspoke airline network under Sichuan Airlines having approximately 254 daily flights. The WTBAM was implemented in C++ using CPLEX 12.3 on a Lenovo notebook with a 2.16$\mathrm{GHz}$ processor. As the arrival time of the disrupted flight was $02: 30$ on the next day and recovery time interval should contain all the flight schedule, we set the schedule recovery from 07:00 to 03:00 on the next day and the time band to $5 \mathrm{~min}$ in order to reduce the gap bound. Then, 1-4 aircraft having mechanical problems or some issue prohibiting them from use between 07:00 to 03:00 on the next day were randomly generated; these events were labelled scenarios 1-4. Combined with the experience of AOCC, the simplex group cycle should not be very large but should not be very small, 60 aircraft in the actual data, roughly divided into 6-15 group. If the simplex group cycle is very large, it may lead to the fact that the calculation time of optimal solution is too long and the potential disruption scope is too large, while if it is too small it may lead to a large potential disruption depth. So we obtain the 7,12 , and 15 three simplex group cycles by setting $m_{0}=5,10,12$ in the $S$ Algorithm. Subsequently, three simplex group cycle partitions are listed in Tables 7-9, and due to the limit of table length, only apart results of WTBAM under these partitions and different disruption thresholds are listed in Tables 12-15. In addition, the results for the simplex group cycle partitions, the scenarios, and the weighted values are presented in Figure 2.

Note that DT 60, DT 30, DT 15, and DT 0 indicate that the flight delay time is more than $60 \mathrm{~min}$, between $30 \mathrm{~min}$ and $60 \mathrm{~min}$, between $15 \mathrm{~min}$ and $30 \mathrm{~min}$, and between $0 \mathrm{~min}$ and $15 \mathrm{~min}$, respectively. Further, GTSN time (s) represents the time required to generate the time-space network in seconds, while CPU time represents the WTBAM calculation time.

Comparison of the data in Tables 12-15 leads to the conclusion that the potential disruption scope $F_{s}$, GTSN time, and CPU time increase with increased $\alpha_{0}$. Subsequently, by the results in Tables $12-15$, we can obtain that GTSN time, CPU time, and potential disruption scope $F_{s}$ satisfy the function in Table 11 and Figure 3. With the increase of $F_{s}$, GTSN time and CPU time are exponential growth and the function relationship is $10^{a x+b}, a>0, b \in R, R$ is the real set. In view of this test, GTSN time and CPU time of second simplex group cycle partitions are generally better than the other simplex group cycle partitions. Note that GTSNij and CPUij indicate the function of GTSN time and potential disruption scope $F_{s}$ in the case of scenario $i$ and $j$ th simplex group cycle partitions, $i=1,2,3,4$ and $j=1,2,3$.

However, the optimal integer solution primarily decreases with increased $\alpha_{0}$, and the gap between the optimal integer and the optimization solution is zero in the examples. Further, when the optimal integer reaches a certain level, it does not necessarily decrease or decreases only slightly, in most cases. These results show that a larger $F_{s}$ is not necessarily 

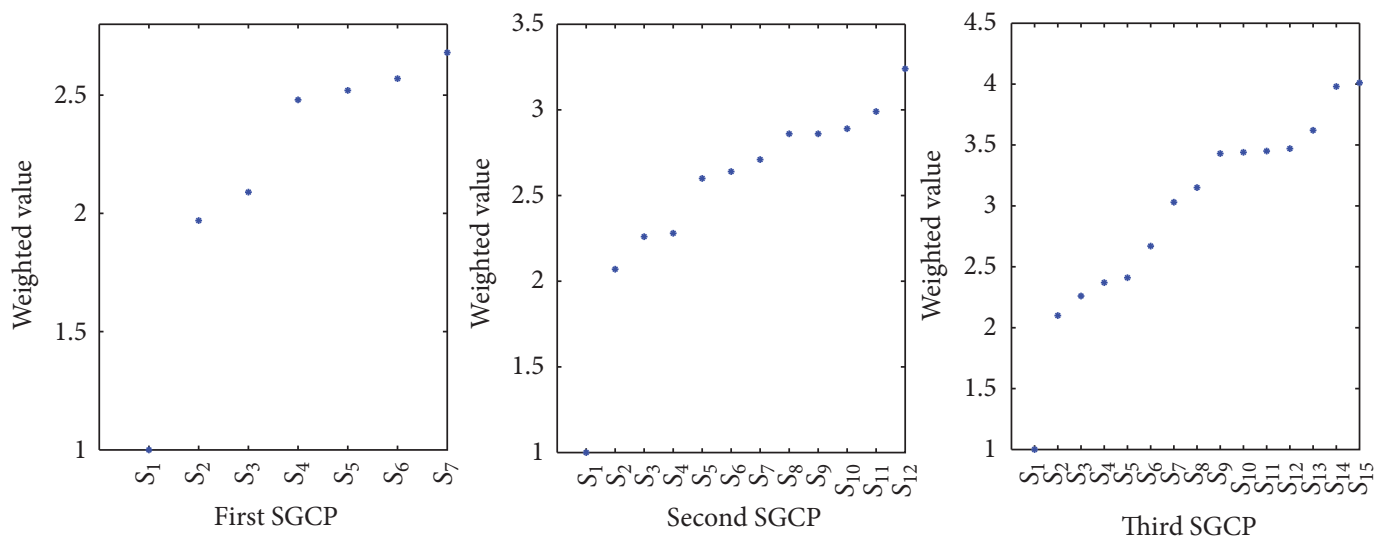

(a) Weighted value under scenario 1
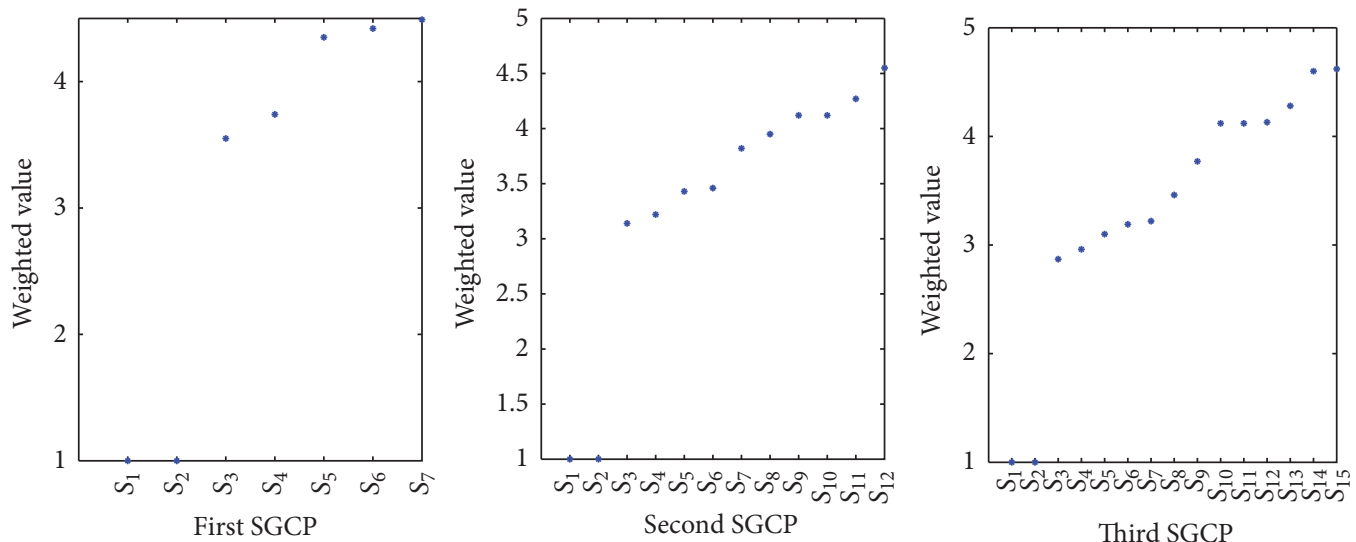

(b) Weighted value under scenario 2
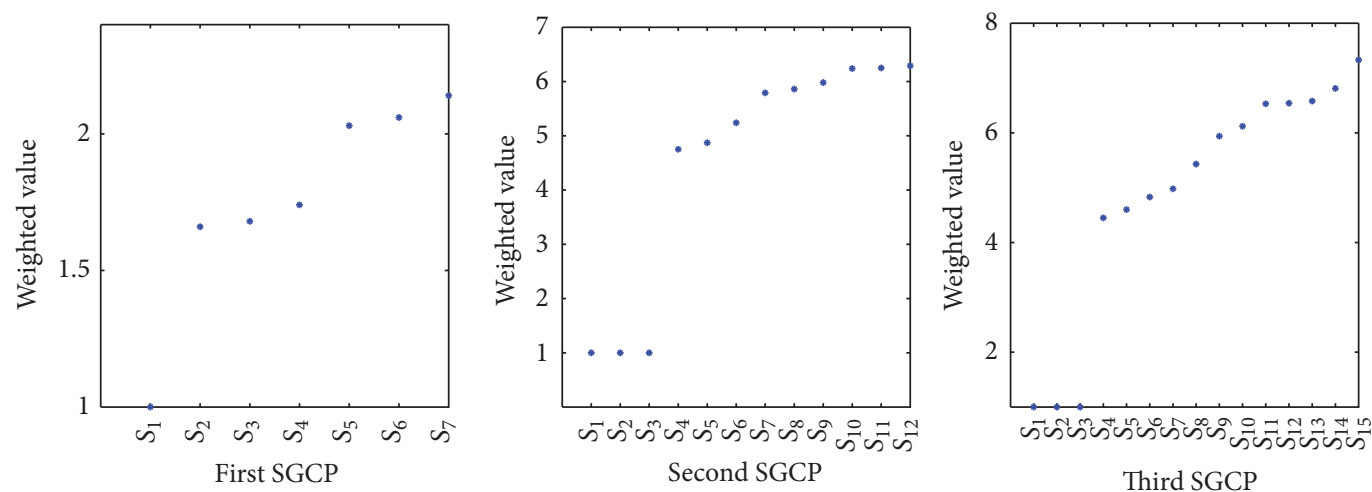

(c) Weighted value under scenario 3
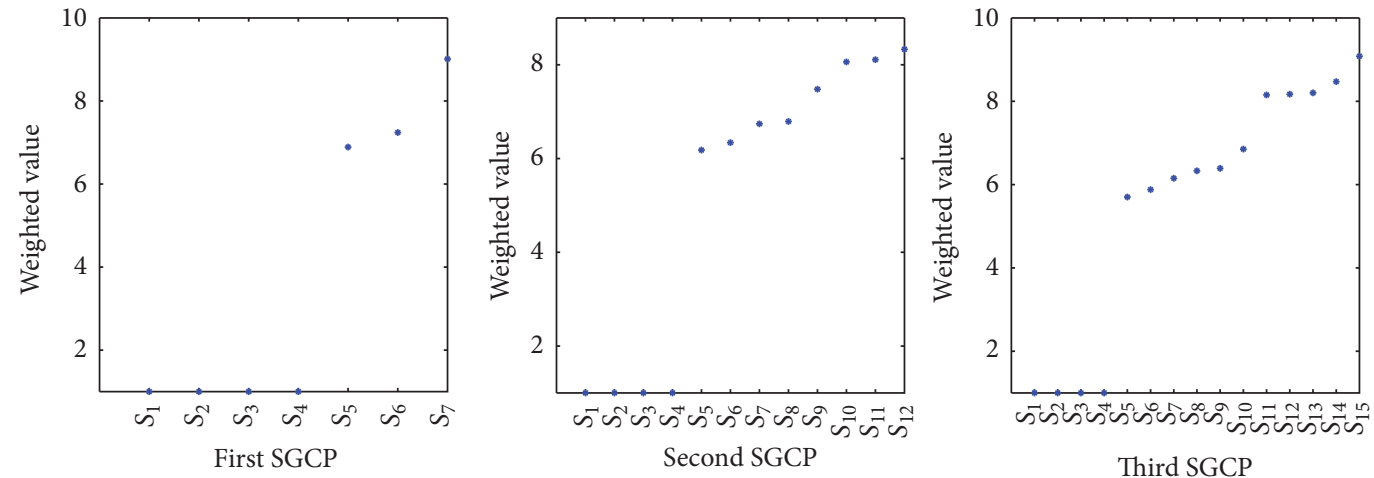

(d) Weighted value under scenario 4

FIGURE 2: Weighted value under different scenarios. 
TABLE 11: Functions for different simplex group cycle partitions and potential disruption scope.

\begin{tabular}{|c|c|c|c|c|c|c|}
\hline & First SGP & Second SGP & Third SGP & First SGP & Second SGP & Third SGP \\
\hline & & Scenario 1 & & & Scenario 2 & \\
\hline GTSN & $10^{2.30 x+0.82}$ & $10^{2.97 x-0.01}$ & $10^{3.28 x+0.03}$ & $10^{2.53 x+0.55}$ & $10^{2.53 x+0.16}$ & $10^{2.98 x+0.18}$ \\
\hline \multirow[t]{2}{*}{$\mathrm{CPU}$} & $10^{2.20 x+0.99}$ & $10^{2.76 x+0.26}$ & $10^{2.97 x+0.34}$ & $10^{2.43 x+0.72}$ & $10^{2.33 x+0.44}$ & $10^{2.74 x+0.47}$ \\
\hline & & Scenario 3 & & & Scenario 4 & \\
\hline GTSN & $10^{2.26 x+0.79}$ & $10^{1.82 x+1.16}$ & $10^{2.56 x+0.51}$ & $10^{2.90 x+0.08}$ & $10^{2.27 x+0.47}$ & $10^{2.60 x+0.41}$ \\
\hline $\mathrm{CPU}$ & $10^{2.12 x+1.02}$ & $10^{1.76 x+1.29}$ & $10^{2.39 x+0.74}$ & $10^{2.73 x+0.33}$ & $10^{2.12 x+0.73}$ & $10^{2.45 x+0.64}$ \\
\hline
\end{tabular}

$x: F_{s}$.

TABLE 12: Results for different simplex group cycle partitions and weighted thresholds under scenario 1.

\begin{tabular}{|c|c|c|c|c|c|c|c|c|c|c|c|c|c|c|c|}
\hline \multirow{2}{*}{$\alpha_{0}$} & \multicolumn{5}{|c|}{ First simplex group cycle partition } & \multicolumn{5}{|c|}{ Second simplex group cycle partition } & \multicolumn{5}{|c|}{ Third simplex group cycle partition } \\
\hline & 1 & 2.1 & 2.5 & 2.6 & 2.7 & 1 & 2.27 & 2.7 & 2.87 & 3.3 & 1 & 2.5 & 3.1 & 3.2 & 4.1 \\
\hline$F_{s}$ & $16.5 \%$ & $38.1 \%$ & $63.3 \%$ & $88.1 \%$ & $100 \%$ & $8.6 \%$ & $22.4 \%$ & $46.8 \%$ & $75.9 \%$ & $100 \%$ & $7.0 \%$ & $24.8 \%$ & $42.9 \%$ & $50.3 \%$ & $100 \%$ \\
\hline DT 60 & 6 & 6 & 8 & 6 & 6 & 3 & 2 & 0 & 0 & 0 & 3 & 2 & 0 & 0 & 0 \\
\hline DT 30 & 1 & 1 & 0 & 0 & 0 & 7 & 8 & 9 & 6 & 6 & 7 & 8 & 6 & 3 & 3 \\
\hline DT 15 & 1 & 1 & 0 & 0 & 0 & 1 & 1 & 0 & 0 & 0 & 1 & 1 & 2 & 1 & 1 \\
\hline DT 0 & 1 & 0 & 1 & 3 & 3 & 1 & 2 & 2 & 3 & 2 & 1 & 3 & 4 & 6 & 6 \\
\hline Nodes & 1953 & 6408 & 11634 & 14314 & 15137 & 679 & 1965 & 4599 & 7091 & 15137 & 489 & 1998 & 5803 & 7331 & 15137 \\
\hline Flight & 3877 & 13903 & 32606 & 44636 & 49439 & 1064 & 3516 & 10331 & 21364 & 49439 & 738 & 3285 & 12309 & 16963 & 49439 \\
\hline Cancellations & 0 & 0 & 0 & 0 & 0 & 0 & 0 & 0 & 0 & 0 & 0 & 0 & 0 & 0 & 0 \\
\hline GTSN time (s) & 7.10 & 101.9 & 403.0 & 677.4 & 801.0 & 0.86 & 7.6 & 45.6 & 134.1 & 816.5 & 0.45 & 8.4 & 86.3 & 144.3 & 797.7 \\
\hline CPU time (s) & 10.9 & 126.1 & 492.3 & 825.0 & 960.6 & 1.9 & 10.8 & 60.2 & 185.2 & 982.4 & 1.3 & 11.2 & 106.7 & 178.8 & 949.7 \\
\hline Optimal integer & 14100 & 13700 & 12447 & 11647 & 11647 & 21500 & 20925 & 15725 & 13510 & 13025 & 21500 & 21391 & 16542 & 13542 & 13040 \\
\hline Gap & $0 \%$ & $0 \%$ & $0 \%$ & $0 \%$ & $0 \%$ & $0 \%$ & $0 \%$ & $0 \%$ & $0 \%$ & $0 \%$ & $0 \%$ & $0 \%$ & $0 \%$ & $0 \%$ & $0 \%$ \\
\hline
\end{tabular}

better. In fact, the real extent of the flight disruption decreases with increased $\alpha_{0}$; however, note that this factor, disruption scope, and depth do increase with increased $\alpha_{0}$ in some cases. Further, the nodes and flight arcs increase with increased $\alpha_{0}$, along with the CPLEX 12.3 computational time.

The AOCC controllers can choose the value of $\alpha_{0}$ by considering the balance between the real flight disruption scope and depth, the CPU time, and the optimal integer value. Subsequently, we first introduce the feasible method to choose the value of $\alpha_{0}$ (Choose Method) in the following. The AOCC controllers choose the appropriate value of $\alpha_{0}$ which should be consistent with the four rules in the following.

\section{Choose Method}

The first rule is to meet the time limit for flight operations recovery.

The second rule is about the flight recovery value in the following formula.

Let $f^{*}$ be $\min \left\{f_{1}^{*}, f_{2}^{*}\right\}, \alpha_{0}^{*}$ be the corresponding threshold of $f^{*}$, and $\alpha_{0}^{i}$ be the corresponding threshold of $f_{i}^{*}, i=1,2$.

$$
\alpha_{0}= \begin{cases}\alpha_{0}^{*} & \frac{\left|f_{1}^{*}-f_{2}^{*}\right|}{f^{*}}>\gamma, \\ \alpha_{0}^{1} \text { or } \alpha_{0}^{2} & \text { otherwise. }\end{cases}
$$

The third rule is as small as possible to the impact of disruption flights scope and depth.

The fourth rule is to ensure the uniqueness flight recovery plan.

If there are two flight recovery plans to meet the previous three rules, then select a smaller flight recovery value. And if the flight recovery value is also the same, then select the CPU time which is less than that of the flight recovery plan.

In this paper, we choose $\gamma=0.25$ which means that the two flight recovery plans can be considered as equivalent when the difference ratio of the two flight recovery values is less than or equal to 0.25 , because the AOCC controllers are not just considering about flight recovery values. The airlines can adjust $\gamma$ to meet their management objectives. Subsequently, we provide a feasible method to measure the impact of disruption flights scope and depth according to the actual situation in China. Firstly, the weighted value of common delay time band is determined by the AOCC controllers, and then we calculate the total impact value. The delay time band and the weighted value are given out in Table 10. In particular, the weighted value can be adjusted according to the requirements of the airlines.

By the Choose Method, we give out the value of $\alpha_{0}$ under different scenarios for the time limit of 5 minutes, 10 minutes, 15 minutes, and 20 minutes in Table 16 . The solution 
TABLE 13: Results for different simplex group cycle partitions and weighted threshold under scenario 2.

\begin{tabular}{|c|c|c|c|c|c|c|c|c|c|c|c|c|c|c|c|}
\hline \multirow{2}{*}{$\alpha_{0}$} & \multicolumn{5}{|c|}{ First simplex group cycle partition } & \multicolumn{5}{|c|}{ Second simplex group cycle partition } & \multicolumn{5}{|c|}{ Third simplex group cycle partition } \\
\hline & 1 & 3.6 & 4.4 & 4.45 & 4.5 & 1 & 3.2 & 3.5 & 3.9 & 4.6 & 1 & 3.0 & 3.3 & 4.2 & 4.7 \\
\hline$F_{s}$ & $28.3 \%$ & $35.8 \%$ & $75.1 \%$ & $89.3 \%$ & $100 \%$ & $12.9 \%$ & $21.6 \%$ & $45.2 \%$ & $81.4 \%$ & $100 \%$ & $12.9 \%$ & $23.6 \%$ & $37.7 \%$ & $79.1 \%$ & $100 \%$ \\
\hline DT 60 & 7 & 7 & 7 & 7 & 7 & 7 & 6 & 8 & 8 & 8 & 7 & 9 & 8 & 8 & 8 \\
\hline DT 30 & 1 & 1 & 1 & 1 & 1 & 0 & 0 & 0 & 0 & 0 & 0 & 0 & 0 & 0 & 0 \\
\hline DT 15 & 1 & 1 & 1 & 1 & 1 & 0 & 0 & 0 & 0 & 0 & 4 & 0 & 0 & 0 & 0 \\
\hline DT 0 & 1 & 1 & 2 & 2 & 2 & 0 & 0 & 2 & 2 & 2 & 0 & 1 & 2 & 2 & 2 \\
\hline Nodes & 2853 & 3479 & 12739 & 14188 & 15158 & 781 & 2261 & 4639 & 5164 & 15158 & 781 & 2353 & 4390 & 12921 & 15158 \\
\hline Flight & 5948 & 7659 & 36361 & 45118 & 49543 & 1369 & 4436 & 10646 & 12089 & 49543 & 1369 & 4984 & 9171 & 37809 & 49543 \\
\hline Cancellations & 1 & 1 & 1 & 1 & 1 & 1 & 1 & 1 & 1 & 1 & 1 & 1 & 1 & 1 & 1 \\
\hline GTSN time (s) & 16.5 & 26.1 & 504.3 & 689.1 & 809.8 & 1.3 & 10.0 & 46.8 & 59.4 & 809.6 & 1.3 & 11.7 & 46.3 & 530.7 & 810.9 \\
\hline CPU time (s) & 23.7 & 35.1 & 606.9 & 841.6 & 992.7 & 3.0 & 14.2 & 62.0 & 78.2 & 1006.5 & 3.1 & 17.5 & 59.6 & 652.9 & 998.4 \\
\hline Optimal integer & 71200 & 61100 & 59835 & 59835 & 59835 & 101300 & 92600 & 63343 & 59743 & 59743 & 101300 & 63000 & 61722 & 60822 & 59722 \\
\hline Gap & $0 \%$ & $0 \%$ & $0 \%$ & $0 \%$ & $0 \%$ & $0 \%$ & $0 \%$ & $0 \%$ & $0 \%$ & $0 \%$ & $0 \%$ & $0 \%$ & $0 \%$ & $0 \%$ & $0 \%$ \\
\hline
\end{tabular}

TABLE 14: Results for different simplex group cycle partitions and weighted threshold under scenario 3.

\begin{tabular}{|c|c|c|c|c|c|c|c|c|c|c|c|c|c|c|c|}
\hline \multirow{2}{*}{$\alpha_{0}$} & \multicolumn{5}{|c|}{ First simplex group cycle partition } & \multicolumn{5}{|c|}{ Second simplex group cycle partition } & \multicolumn{5}{|c|}{ Third simplex group cycle partition } \\
\hline & 1 & 1.7 & 1.8 & 2.1 & 2.2 & 1 & 4.8 & 4.9 & 5.9 & 6.3 & 1 & 4.5 & 6.0 & 6.6 & 7.4 \\
\hline$F_{s}$ & $25.1 \%$ & $49.2 \%$ & $63.3 \%$ & $88.1 \%$ & $100 \%$ & $27.1 \%$ & $35.8 \%$ & $40.9 \%$ & $65.3 \%$ & $100 \%$ & $22.0 \%$ & $29.1 \%$ & $58.6 \%$ & $87.7 \%$ & $100 \%$ \\
\hline DT 60 & 10 & 6 & 5 & 5 & 5 & 6 & 4 & 5 & 5 & 3 & 18 & 16 & 11 & 6 & 5 \\
\hline DT 30 & 4 & 7 & 8 & 8 & 8 & 4 & 1 & 1 & 1 & 2 & 1 & 1 & 2 & 2 & 2 \\
\hline DT 15 & 2 & 3 & 4 & 4 & 4 & 1 & 1 & 1 & 1 & 1 & 2 & 2 & 3 & 4 & 2 \\
\hline DT 0 & 6 & 7 & 6 & 6 & 6 & 5 & 5 & 5 & 5 & 7 & 4 & 6 & 7 & 10 & 11 \\
\hline Nodes & 2951 & 5420 & 11407 & 14052 & 14879 & 3530 & 5200 & 5688 & 10177 & 19 & 1803 & 2798 & 8566 & 13603 & 14879 \\
\hline Flight arcs & 6474 & 14395 & 31950 & 43790 & 48559 & 7786 & 11783 & 13309 & 28117 & 48559 & 4070 & 6430 & 22541 & 43039 & 48559 \\
\hline Cancellations & 2 & 2 & 2 & 2 & 2 & 4 & 4 & 4 & 2 & 2 & 2 & 2 & 2 & 2 & 2 \\
\hline GTSN time (s) & 17.5 & 68.4 & 395.2 & 659.4 & 781.7 & 33.0 & 71.1 & 89.4 & 324.7 & 781.0 & 8.0 & 17.9 & 224.5 & 624.9 & 777.1 \\
\hline CPU time (s) & 27.5 & 97.2 & 497.5 & 807.4 & 957.4 & 44.4 & 91.8 & 112.9 & 409.4 & 938.8 & 13.9 & 25.7 & 278.9 & 766.8 & 942.2 \\
\hline Optimal integer & 89500 & 76071 & 73850 & 73850 & 73850 & 101299 & 93500 & 88900 & 81879 & 74580 & 127700 & 108000 & 105654 & 90300 & 87500 \\
\hline Gap & $0 \%$ & $0 \%$ & $0 \%$ & $0 \%$ & $0 \%$ & $0 \%$ & $0 \%$ & $0 \%$ & $0 \%$ & $0 \%$ & $0 \%$ & $0 \%$ & $0 \%$ & $0 \%$ & $0 \%$ \\
\hline
\end{tabular}

TABLE 15: Results for different simplex group cycle partitions and weighted threshold under scenario 4.

\begin{tabular}{|c|c|c|c|c|c|c|c|c|c|c|c|c|c|c|}
\hline \multirow{2}{*}{$\alpha_{0}$} & \multicolumn{4}{|c|}{ First simplex group cycle partition } & \multicolumn{5}{|c|}{ Second simplex group cycle partition } & \multicolumn{5}{|c|}{ Third simplex group cycle partition } \\
\hline & 1 & 6.9 & 7.3 & 9.1 & 1 & 6.2 & 6.75 & 8.1 & 8.4 & 1 & 5.8 & 6.4 & 8.3 & 9.10 \\
\hline$\overline{F_{s}}$ & $66.5 \%$ & $74.0 \%$ & $88.1 \%$ & $100 \%$ & $40.1 \%$ & $48.8 \%$ & $62.5 \%$ & $86.2 \%$ & $100 \%$ & $33.8 \%$ & $40.9 \%$ & $58.6 \%$ & $87.7 \%$ & $100 \%$ \\
\hline DT 60 & 14 & 15 & 16 & 17 & 18 & 21 & 24 & 25 & 29 & 20 & 25 & 25 & 26 & 29 \\
\hline DT 30 & 2 & 2 & 2 & 2 & 8 & 5 & 8 & 5 & 3 & 6 & 6 & 5 & 2 & 2 \\
\hline DT 15 & 2 & 3 & 3 & 2 & 3 & 3 & 2 & 3 & 3 & 3 & 1 & 3 & 2 & 1 \\
\hline DT 0 & 9 & 7 & 8 & 8 & 9 & 8 & 8 & 9 & 7 & 7 & 8 & 9 & 11 & 9 \\
\hline Nodes & 6475 & 7037 & 14034 & 14858 & 2959 & 4200 & 5466 & 7735 & 14858 & 2425 & 3474 & 6503 & 13585 & 14858 \\
\hline Flight arcs & 19023 & 20974 & 43699 & 48454 & 8040 & 11335 & 15674 & 25801 & 48454 & 6326 & 8982 & 16996 & 42950 & 48454 \\
\hline Cancellations & 3 & 3 & 3 & 3 & 3 & 3 & 3 & 3 & 3 & 3 & 3 & 3 & 3 & 3 \\
\hline GTSN time (s) & 108.6 & 131.3 & 662.4 & 783.9 & 23.8 & 45.1 & 79.8 & 175.1 & 781.7 & 15.6 & 29.9 & 125.4 & 625.3 & 777.6 \\
\hline CPU time (s) & 150.2 & 184.2 & 824.4 & 977.9 & 38.2 & 65.6 & 110.3 & 259.3 & 923.2 & 24.7 & 41.9 & 158.6 & 769.7 & 931.7 \\
\hline Best integer & 140700 & 140700 & 137399 & 137399 & 172300 & 150500 & 144374 & 142174 & 139773 & 182000 & 155700 & 149239 & 143391 & 141991 \\
\hline Gap & $0 \%$ & $0 \%$ & $0 \%$ & $0 \%$ & $0 \%$ & $0 \%$ & $0 \%$ & $0 \%$ & $0 \%$ & $0 \%$ & $0 \%$ & $0 \%$ & $0 \%$ & $0 \%$ \\
\hline
\end{tabular}


TABLE 16: Choose $\alpha_{0}$ for different time limit under different scenarios.

\begin{tabular}{|c|c|c|c|c|c|c|c|c|c|c|c|c|c|c|c|c|}
\hline \multirow{2}{*}{$\begin{array}{l}\text { Time limit } \\
\text { Scenario }\end{array}$} & \multicolumn{4}{|c|}{$5 \mathrm{~min}$} & \multicolumn{4}{|c|}{$10 \mathrm{~min}$} & \multicolumn{4}{|c|}{$15 \mathrm{~min}$} & \multicolumn{4}{|c|}{$20 \mathrm{~min}$} \\
\hline & $S_{1}$ & $S_{2}$ & $S_{3}$ & $S_{4}$ & $S_{1}$ & $S_{2}$ & $S_{3}$ & $S_{4}$ & $S_{1}$ & $S_{2}$ & $S_{3}$ & $S_{4}$ & $S_{1}$ & $S_{2}$ & $S_{3}$ & $S_{4}$ \\
\hline$\alpha_{0}$ & 3.2 & 3.6 & 4.9 & 1 & 3.2 & 3.6 & 5.9 & 1 & 3.2 & 3.6 & 5.9 & 1 & 3.2 & 3.6 & 6.3 & 1 \\
\hline SGP & 3 & 1 & 2 & 1 & 3 & 1 & 2 & 1 & 3 & 1 & 2 & 1 & 3 & 1 & 2 & 1 \\
\hline $\begin{array}{l}\text { CPU time } \\
\text { (s) }\end{array}$ & 178.8 & 35.1 & 112.9 & 150.2 & 178.8 & 35.1 & 409.4 & 150.2 & 178.8 & 35.1 & 409.4 & 150.2 & 178.8 & 35.1 & 938.8 & 150.2 \\
\hline Impact & 20 & 57 & 73 & 129 & 20 & 57 & 57 & 129 & 20 & 57 & 57 & 129 & 20 & 57 & 51 & 129 \\
\hline $\begin{array}{l}\text { Optimal } \\
\text { integer }\end{array}$ & 13542 & 61100 & 88900 & 140700 & 13542 & 61100 & 81879 & 140700 & 13542 & 61100 & 81879 & 140700 & 13542 & 61100 & 74580 & 140700 \\
\hline
\end{tabular}
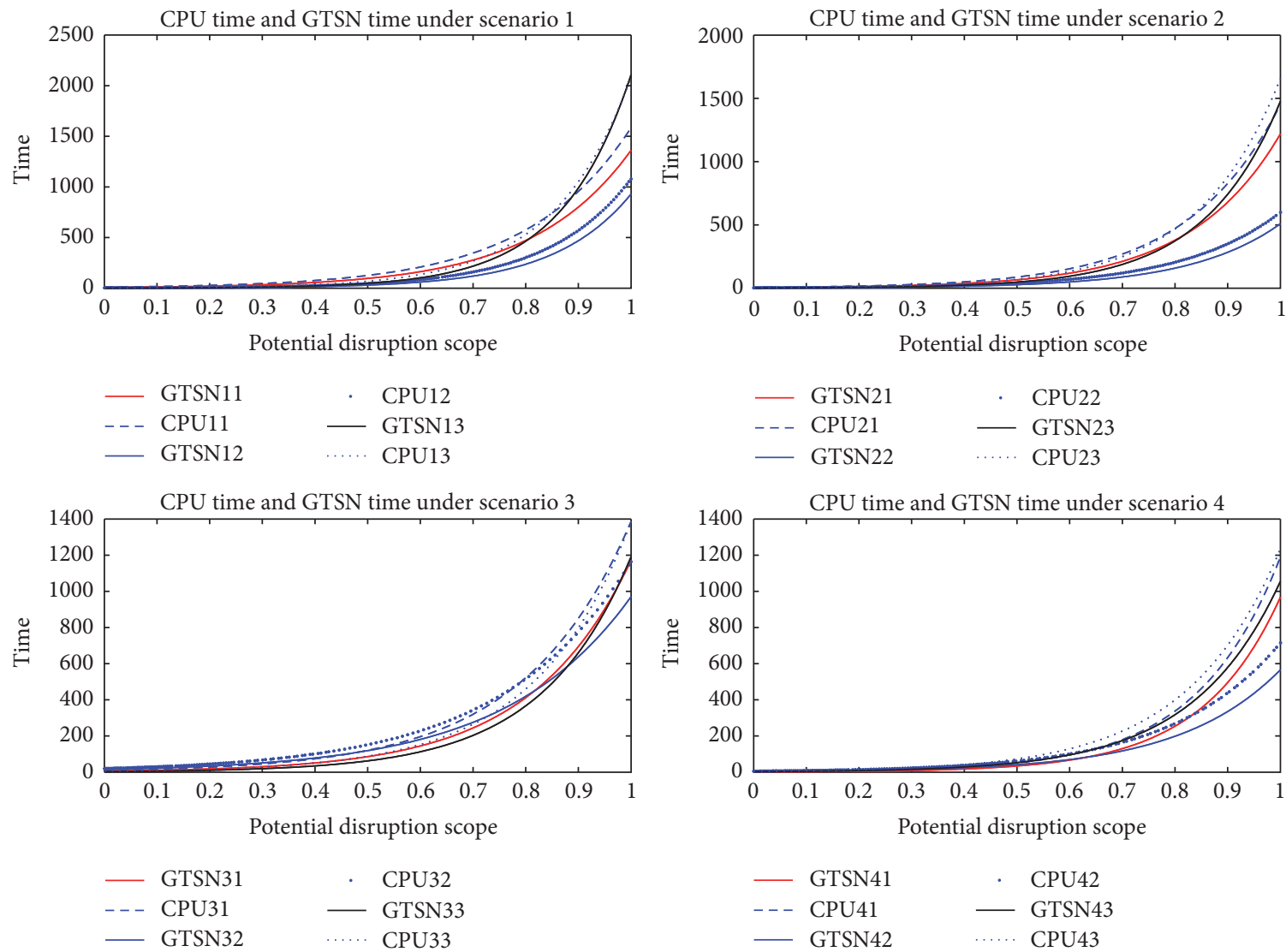

FIgURE 3: CPU Ttme and GTSN time under different scenarios.

is obtained considering the flight disruption scope and depth, computation time, and recovery value under some appropriate rules, which is generally different from the minimum recovery value.

Note that the time-space network generation time occupies the majority of the computational time required to solve the WTBAM. In fact, the feasible region of the WTBAM is the time-space network, the total CPU time primarily consists of the time required to generated a feasible region, and the well-ordered property requirement must be satisfied or approximately satisfied in most cases.

By comparing the data for the same disruption scenario, we note that the simplex group cycle partition is finer, the weighted value is larger, and the optimal integer value is largest for all potential flight disruptions. The number of aircraft decreases as the number of simplex group cycles increases, according to Tables 7-9. For the same disruption scenario, the weighted value also increases when the number of simplex group cycles increases, according to Figure 2.

\section{Conclusions and Discussion}

In this study, we constructed the weighted time-band approximation model (WTBAM) incorporating simplex group cycles for flight operations recovery. The objective of the WTBAM as regards flight operations recovery is to minimize both the delay costs and the cancellation costs through implementation of a weighted threshold. The series of experiments conducted here has shown that the potential disruption scope and depth, computation time, and recovery value are 
controlled by the disruption threshold. Considering the balance between the real impact of flight disruption scope and depth, the CPU operation time, and the recovery value, airline operations control center (AOCC) controllers can conveniently obtain satisfactory recovery plan by changing the disruption threshold. Through examination of the WTBAM characteristics, it was determined that the gap between the linear programming relaxation of the WTBAM (LPRWTBAM) and WTBAM solutions is zero under some conditions. Therefore, the total CPU operation time is primarily comprised of the time required for the WTBAM to generate a feasible region for the time-space network. The computation time decreases as the disruption threshold is decreased. In future research, methods to partition the flights or aircraft into the optimal simplex group cycles will be investigated.

\section{Nomenclature}

\section{Indices}

$$
\begin{array}{ll}
i, j: & \text { Node indices } \\
k: & \text { Flight index } \\
s: & \text { Simplex group cycle index. }
\end{array}
$$

Sets

$S F_{s}: \quad$ Set of flights in simplex group cycle $s$

$S A_{s}$ : $\quad$ Set of aircraft in simplex group cycle $s$

$\left|S A_{s}\right|: \quad$ Number of aircraft in $S A_{s}$

$G(i)$ : $\quad$ Set of flights originating at station-time node $i$

$H(k, i)$ : Set of destination nodes for flight $k$ originating at station-time node $i$

I: $\quad$ Set of station-time nodes

$J: \quad$ Set of station-sink nodes

$S^{*}$ : $\quad$ Set containing simplex group cycle $s$, with at least one schedule disruption in $s$

$S: \quad$ Set of all simplex group cycles

$W: \quad$ Set of all weighted values $=\left\{W_{s}, s \in S\right\}$

$N(i)$ : Set of flights terminating at node $i \in J$

$M(k, i)$ : Set of origin station-time nodes for flight $k$ terminating at node $i \in J$

$P(k)$ : Set of station-time nodes from which flight $k$ originates

$Q(i): \quad$ Set of station-time nodes terminating at station-sink node $i \in J$

$\operatorname{PDFS}^{\alpha_{0}}$ : Set of potential disrupted flights determined by Algorithm 2.

\section{Parameters}

$a_{i}^{\alpha_{0}}$ : Number of aircraft executing $P D F S^{\alpha_{0}}$ flights that become available at station-time node $i$ at time zero

$c_{k}$ : Cost of cancelling flight $k$

$w^{k}$ : Weighted value of flight $k$

$d_{i j}^{k}$ : Delay cost of flight $k$ from station-time node $i$ to node $j$

$w_{i j}^{k}$ : Weighted value of flight $k$ from station-time node $i$ to node $j$

$h_{i}^{\alpha_{0}}$ : Number of aircraft that execute $P D F S^{\alpha_{0}}$ flights required to terminate at station-sink node $i \in J$.
Variables

$x_{i j}^{k}$ : Magnitude of aircraft flow for flight $k$ from station-time node $i$ to node $j$

$y_{k}$ : Cancellation indicator for flight $k$

$z_{i}$ : Magnitude of aircraft flow required to execute $P D F S^{\alpha_{0}}$ flights from station-time node $i$ to station-sink node at same station.

\section{Competing Interests}

The authors declare that there are no competing interests regarding the publication of this manuscript.

\section{Acknowledgments}

Haiwen Xu was supported by the Joint Fund of the National Natural Science Foundation of China and the Civil Aviation Administration of China (Grant no. U1233105).

\section{References}

[1] P. Belobaba, A. Odoni, and C. Barnhart, The Global Airline Industry, John Wiley \& Sons, Chippenham, Wiltshire, 2009.

[2] D. Teodorović and S. Guberinić, "Optimal dispatching strategy on an airline network after a schedule perturbation," European Journal of Operational Research, vol. 15, no. 2, pp. 178-182, 1984.

[3] D. Teodorović and G. Stojković, "Model for operational daily airline scheduling," Transportation Planning and Technology, vol. 14, no. 4, pp. 273-285, 1990.

[4] A. I. Z. Jarrah, G. Yu, N. Krishnamurthy, and A. Rakshit, "Decision support framework for airline flight cancellations and delays," Transportation Science, vol. 27, no. 3, pp. 266-280, 1993.

[5] G. Yu, "An optimization model for airlines' irregular operation system control," in Proceedings of the International Symposium on Operations Research with Applications in Engineering, Technology, and Management, Beijing, China, 1995.

[6] S. Yan and D.-H. Yang, "A decision support framework for handling schedule perturbation," Transportation Research Part B: Methodological, vol. 30, no. 6, pp. 405-419, 1996.

[7] S. Yan and C.-G. Lin, "Airline scheduling for the temporary closure of airports," Transportation Science, vol. 31, no. 1, pp. 7282, 1997.

[8] S. Yan and Y.-P. Tu, "Multifleet routing and multistop flight scheduling for schedule perturbation," European Journal of Operational Research, vol. 103, no. 1, pp. 155-169, 1997.

[9] J.-M. Cao and A. Kanafani, "Real-time decision support for integration of airline flight cancellations and delays, part I: mathematical formulation," Transportation Planning and Technology, vol. 20, no. 3, pp. 183-199, 1997.

[10] J.-M. Cao and A. Kanafani, "Real-time decision support for integration of airline flight cancellations and delays, part II: algorithm and computational experiments," Transportation Planning and Technology, vol. 20, no. 3, pp. 201-217, 1997.

[11] G. Yu, Operations Research in the Airline Industry, Kluwer Academic Publishers, 1997.

[12] J. F. Bard, G. Yu, and M. F. Argüello, "Optimizing aircraft routings in response to groundings and delays," IIE Transactions (Institute of Industrial Engineers), vol. 33, no. 10, pp. 931-947, 2001. 
[13] S. Bratu and C. Barnhart, "Flight operations recovery: new approaches considering passenger recovery," Journal of Scheduling, vol. 9, no. 3, pp. 279-298, 2006.

[14] Y. Hu, B. Xu, J. F. Bard, H. Chi, and M. Gao, "Optimization of multi-fleet aircraft routing considering passenger transiting under airline disruption," Computers \& Industrial Engineering, vol. 80, pp. 132-144, 2015.

[15] X. L. Zhao, J. F. Zhu, and M. Guo, "Study on modelling and algorithm of irregular flight delay operation," Systems EngineeringTheory and Practice, vol. 4, pp. 129-134, 2008.

[16] S. J. Maher, "A novel passenger recovery approach for the integrated airline recovery problem," Computers and Operations Research, vol. 57, pp. 123-137, 2015.

[17] K. Sinclair, J.-F. Cordeau, and G. Laporte, "Improvements to a large neighborhood search heuristic for an integrated aircraft and passenger recovery problem," European Journal of Operational Research, vol. 233, no. 1, pp. 234-245, 2014.

[18] D. Mou and W. Zhao, "An irregular flight scheduling model and algorithm under the uncertainty theory," Journal of Applied Mathematics, vol. 2013, Article ID 361926, 8 pages, 2013.

[19] A. J. M. Castro, A. P. Rocha, and E. Oliveira, A New Approach for Disruption Management in Airline Operations Control, vol. 562 of Springer Studies in Computational Intelligence, Springer, 2014.

[20] M. F. Argüello, J. F. Bard, and G. Yu, "A GRASP for aircraft routing in response to groundings and delays," Journal of Combinatorial Optimization, vol. 1, no. 3, pp. 211-228, 1997.

[21] M. Clarke, "Development of heuristic procedures for flight rescheduling in the aftermath of irregular airline operations," Working Paper, 1997.

[22] B. G. Thengvall, G. Yu, and J. F. Bard, "Multiple fleet aircraft schedule recovery following hub closures," Transportation Research Part A: Policy and Practice, vol. 35, no. 4, pp. 289-308, 2001.

[23] M. Løve, K. Sørensen, J. Larsen, and J. Clausen, "Using heuristics to solve the dedicated aircraft recovery problem," Central European Journal of Operations Research, vol. 13, pp. 189-207, 2005.

[24] S. Bisaillon, J.-F. Cordeau, G. Laporte, and F. Pasin, "A large neighbourhood search heuristic for the aircraft and passenger recovery problem," A Quarterly Journal of Operations Research, vol. 9, no. 2, pp. 139-157, 2011.

[25] K. Sinclair, J.-F. Cordeau, and G. Laporte, "Improvements to a large neighborhood search heuristic for an integrated aircraft and passenger recovery problem," European Journal of Operational Research, vol. 233, no. 1, pp. 234-245, 2014.

[26] J. D. Petersen, G. Sölveling, J.-P. Clarke, E. L. Johnson, and S. Shebalov, "An optimization approach to airline integrated recovery," Transportation Science, vol. 46, no. 4, pp. 482-500, 2012.

[27] S. Lan, J.-P. Clarke, and C. Barnhart, "Planning for robust airline operations: optimizing aircraft routings and flight departure times to minimize passenger disruptions," Journal of Transportation Science, vol. 40, no. 1, pp. 15-28, 2006.

[28] H. Xu et al., "The time-band approximation model on flight operations recovery: considering the multi-hub-spoke airlines in China," in Airline Economics and Operations II, Sponsor: Aviation Application, H. Jiang, Ed., Informs International Conference Beijing, Department of Industrial Engineering, Tsinghua University, Beijing, China, 2012.
[29] H. Xu, S. C. Han, Y. Zhang, and J. G. Li, "The time-band approximation model on flight operations recovery model considering random flight ying time in China," in Proceedings of the IEEE International Conference on Systems, Man and Cybernetics (SMC '15): Big Data Analytics for Human-Centric Systems, Hong Kong, 2015.

[30] J. Clausen, A. Larsen, J. Larsen, and N. J. Rezanova, “Disruption management in the airline industry-concepts, models and methods," Computers and Operations Research, vol. 37, no. 5, pp. 809-821, 2010.

[31] J. M. Rosenberger, E. L. Johnson, and G. L. Nemhauser, "A robust fleet-assignment model with hub isolation and short cycles," Transportation Science, vol. 38, no. 3, pp. 357-368, 2004.

[32] M. F. Argüello, J. F. Bard, and G. Yu, "Models and methods for managing airline irregular operations," in Operations Research in the Airline Industry, Y. Gang, Ed., vol. 9, pp. 1-45, Kluwer Academic Publishers, 1998. 


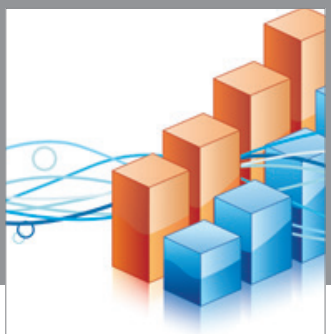

Advances in

Operations Research

vatem alat4

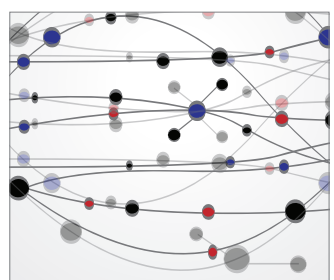

\section{The Scientific} World Journal
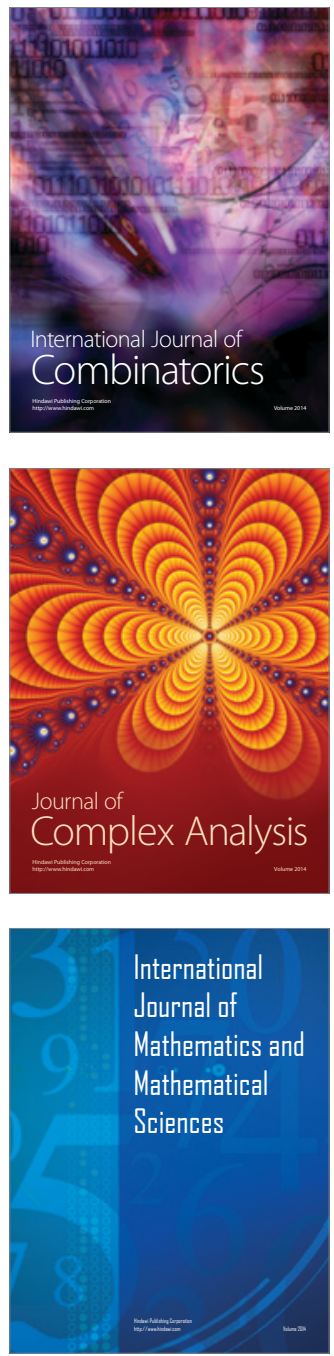
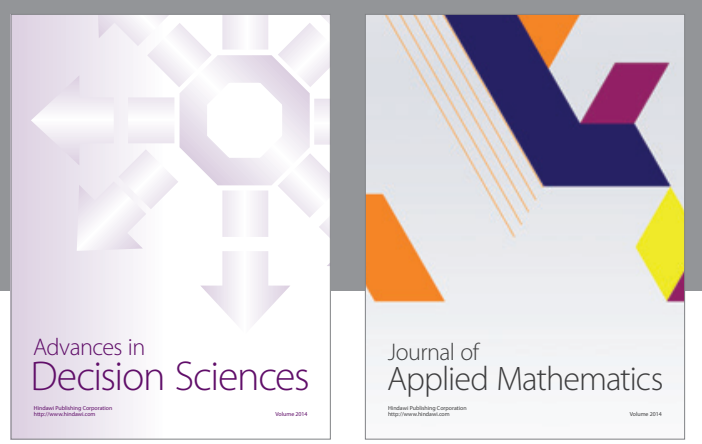

Algebra

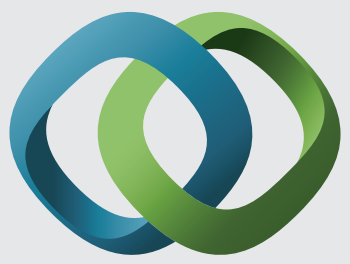

\section{Hindawi}

Submit your manuscripts at

http://www.hindawi.com
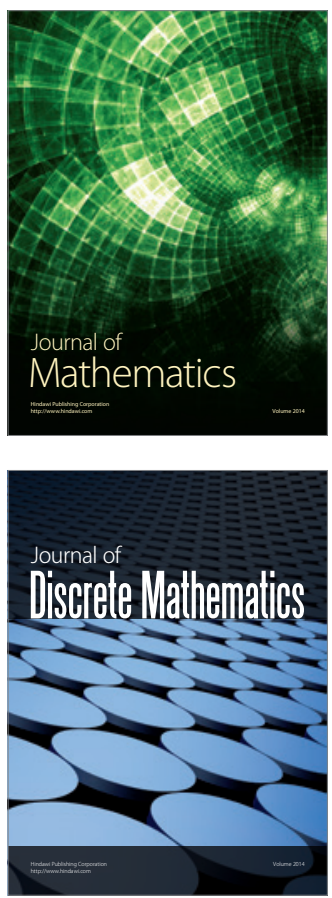

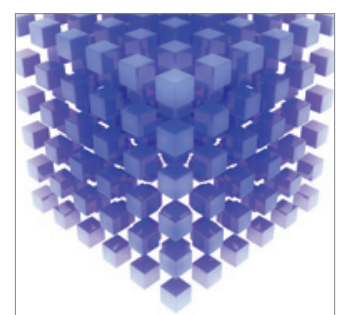

Mathematical Problems in Engineering
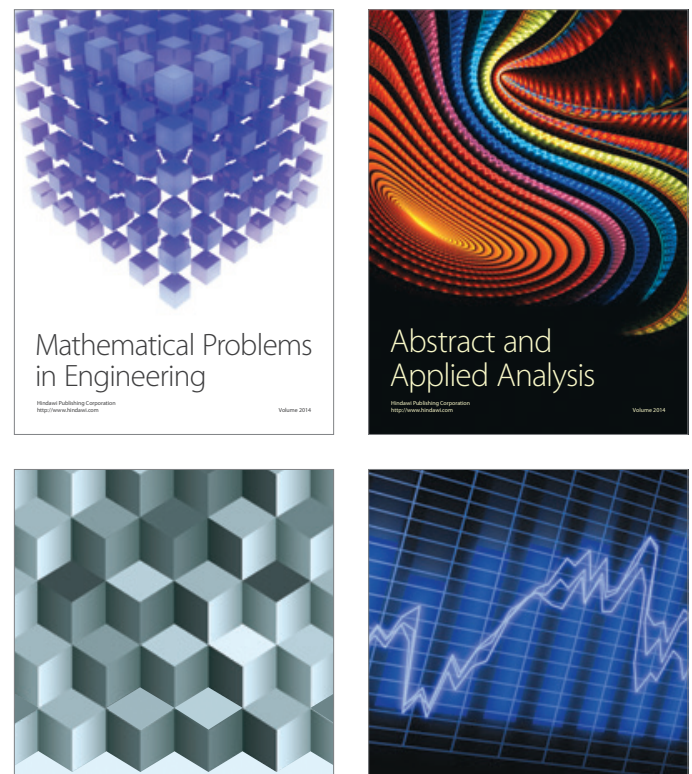

Journal of

Function Spaces

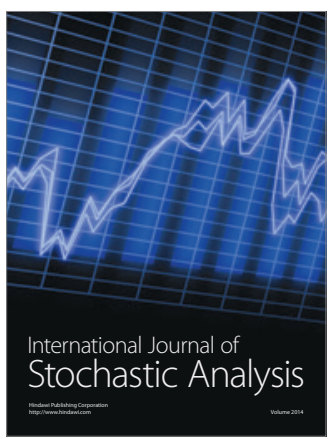

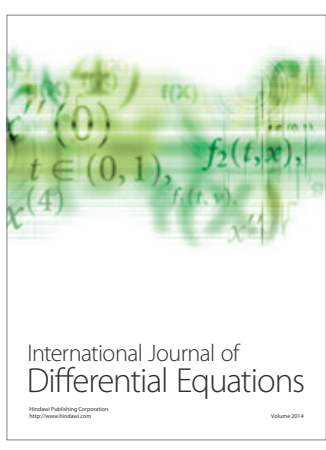
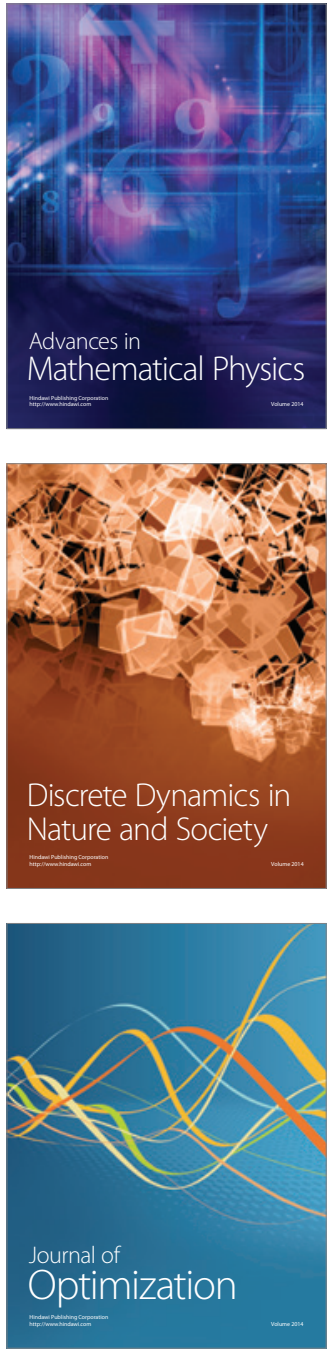